\title{
Low resolution spectroscopy of selected Algol systems
}

\author{
D. Shanti Priya ${ }^{1 *} \cdot$ J. Rukmini ${ }^{1} \cdot$ M. Parthasarathy ${ }^{2}$. \\ D.K. Sahu ${ }^{2}$ Vijay Mohan ${ }^{3}$ B.C. Bhatt ${ }^{2} \cdot$ Vineet S. Thomas $^{4}$
}

\begin{abstract}
The analysis of spectroscopic data for 30 Algoltype binaries is presented. All these systems are short period Algols having primaries with spectral types B and A. Dominant spectral lines were identified for the spectra collected and their equivalent widths were calculated. All the spectra were examined to understand presence of mass transfer, a disk or circumstellar matter and chromospheric emission. We also present first spectroscopic and period study for few Algols and conclude that high resolution spectra within and outside the primary minimum are needed for better understanding of these Algol type close binaries.
\end{abstract}

Keywords Algols; period study; spectral lines; masstransfer

\section{Introduction}

Algol systems are semi-detached close interacting binaries, which consist of a hot and more massive B-A type main-sequence primary star and a cool, giant or subgiant secondary star of F-K spectral type that transfers

D. Shanti Priya

J. Rukmini

M. Parthasarathy

D.K. Sahu

Vijay Mohan

B.C. Bhatt

Vineet S. Thomas

${ }^{1}$ Department of Astronomy, Osmania University, Hyderabad, Telangana 500007, India. *Corresponding author email: astroshanti@gmail.com

${ }^{2}$ Indian Institute of Astrophysics, Koramangala, Bengaluru, Karnataka 560034, India.

${ }^{3}$ Inter-University Centre for Astronomy and Astrophysics, Pune University Campus, Pune 411007, India.

${ }^{4}$ The University of Akron, Akron, Ohio 44304, USA. mass and angular momentum to the primary via Roche lobe overflow (RLOF) Kopal (1955); Giuricin et al. (1983)). They are an important source to study various phenomena, such as mass transfer and accretion, angular momentum, magnetic activity in the late-type companion and orbit evolution. Studies of these binaries resulting in reliable fundamental parameters has significantly increased due to the availability of high-resolution spectroscopic data. Detailed studies of Algols, both detached and semidetached are important in understanding and developing theoretical models representing the formation and evolution of binary systems as well as a single stars. The chemical composition of stellar photospheres in mass-transferring binary systems like Algols is an important diagnostic to study the nucleosynthesis processes which occur deep inside the component stars. This study also provides the information on the components history. The evolutionary process in these interacting binaries cause many observable effects (changes in orbital period, erratic light variability, distorted radial velocity curves, etc.), with the most important being mass transfer. Up to $80 \%$ of the more massive star's initial mass can be lost, exposing deep inner layers of the star that have been changed by thermonuclear fusion during its main sequence evolution. There are also changes happening in some of the material transferred to the companion. The abundance pattern in Algol-type binaries could reveal their past, and would be strong evidence for postulated mass transfer between the components (Sarna and De Greve (1996)). The current work highlights the results of low-resolution spectroscopy carried out over several nights to investigate the changes in emission and absorption line profiles of the star systems. The orbital period variations were investigated for selected Algol-type eclipsing binaries in this study. The O-C diagrams of 13 of these Algols show some measurable period variation, whereas for the other 17 Algols the changes are 
undefined due to insufficient number or non-availability of data.

\section{Observations, Data Reduction and Analysis}

Low resolution spectral observations were carried out using the 2-m Himalayan Chandra Telescope (HCT) of Indian Astronomical Observatory equipped with a $\mathrm{Hi}$ malaya Faint Object Spectrograph Camera (HFOSC). The telescope has a $2 \mathrm{~K} \times 4 \mathrm{~K}$ CCD and a central strip of $500 \mathrm{x}$ 3500 pixels was used. A total of 31 Algols (as listed in Table 1) were observed on various nights in the years 2013 and 2014 with exposures varying between 380s-1200s as listed in Table 1 . The observed data covers wavelength region of 3500-9100 $\AA$. This is achieved using two grism Gr7 and Gr8. For Gr7 the wavelength coverage is 3500$7800 \AA$ and for Gr8 it is $5200-9200 \AA$. This gives a dispersion of $1.5 \AA /$ pixel and a resolution of $\sim 11 \AA$. The FeAr arc lamp was used for wavelength calibration of spectrum taken with Gr7 and FeNe arc lamp spectrum was used for wavelength calibration of spectrum taken with Gr8. The IRAF ${ }^{\mathrm{T}}$ package and different sub-packages like ONEDSPEC were used for reducing the spectra after bias and flat-field corrections. Later the spectra were normalized for further studies.

\section{Period Analysis and Spectroscopic study}

In order to carry out period analysis for the Algols in this study, times of minimum available in the literature were collected and the new phases listed in Table 1 were calculated. Fig 1 show the $(\mathrm{O}-\mathrm{C})$ diagrams that are constructed from all times of primary minima available. Only $13 \mathrm{Al}-$ gols have data which were used to investigate period variations. The $\mathrm{O}-\mathrm{C}$ diagrams show significant period variations which can be fit to polynomial equation. The period changes of some of the systems are tilted sinusoidal variations superimposed on either upward or downward parabolic forms suggesting presence of tertiary component.

Algols are close interacting binaries in which mass transfer occurs as a result of RLOF from the evolved latetype secondary star. The stream, impact point and other accretion structures arising from mass transfer often give rise to emission and absorption lines. In this study we also present the observed spectral profiles of 30 Algol binaries. The dominant spectral lines identified in spectral

${ }^{1}$ IRAF is distributed by the National Optical Astronomy Observatory, which is operated by the Association of Universities for Research in Astronomy (AURA) under cooperative agreement with the National Science Foundation. range $3000-7000 \AA$ and their profiles are presented and discussed. The equivalent widths of identified spectral lines with absorption feature for all the Algols and emission feature for XY Pup are being reported for the first time. The equivalent widths obtained for the dominant spectral lines are given in Table 2 . To derive the spectral class and thus the effective temperatures of the components of Algols in this study, the observed spectra were first compared with standard spectra catalogued in the library of Stellar Spectra (Jacoby et al.(1984)). Based on chi square minima and visual inspections, the best fit spectral model was selected. It was also observed that the dominant spectral lines were normal and free of emission (except in XY Pup) while atomic absorption features matched very well with the corresponding atomic absorption features of the standard spectra.

\subsection{V0616 Aql}

V0616 Aql (= GSC 04147-01115 = TYC 4147-1115-1, V = 14.55 ) is one of the least studied Algol binary which was first catalogued by Hoffmeister (1943) and the linear elements were derived by|Zejda (2002a); Kreiner (2004). This is one of least observed Algol with only 6 times of minima(ToM) in the literature. The O-C residuals were obtained from the recent epoch available in the literature and the same is plotted in the Figure 2. The quadratic fit for the $\mathrm{O}-\mathrm{C}$ variation resulted in $\mathrm{dp} / \mathrm{dt}=-0.001481$ days/yr. The significant change observed from the best fit curve is of that of a decreasing trend extended over a period of 8 years with 200 orbital cycles. The ephemeris obtained in the current study is HJD (Min I) $=2456770.486$ $+1^{d} .690532 \times$ E. However, better conclusions can be laid on only with further observations.

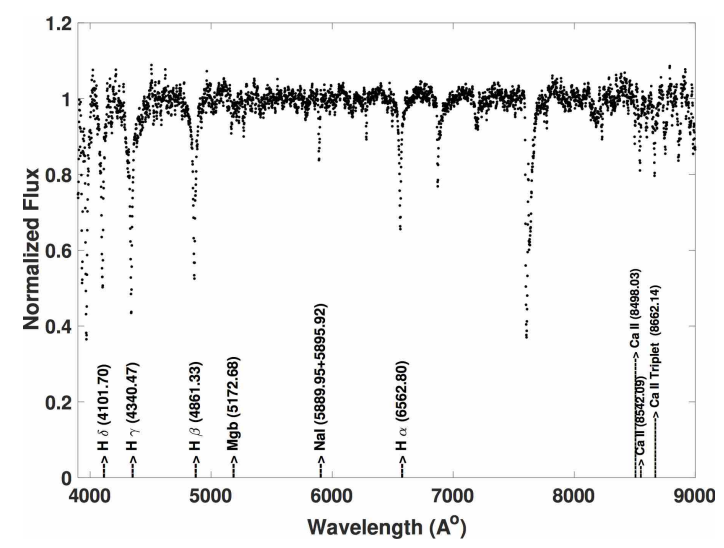

Fig. 1 Spectrum of V0616 Aql.

\footnotetext{
${ }^{2}$ Negative sign indicates emission line strength.
} 
Table 1 Observed Algols: their periods and UT date of observations

\begin{tabular}{|c|c|c|c|c|c|c|}
\hline S.No. & Algol Name & Period & Date of Observation & Epoch & Phase & Exposure time(secs) \\
\hline 1. & V0616 Aql & 1.6906 & Apr 23, 2014 & 2456770.486 & 0.973 & 1380 \\
\hline 2. & V0769 Aql & 4.5623 & Nov 20, 2013 & 2452500.270 & 0.977 & 1200 \\
\hline 3. & V1340 Aql & 1.5969 & Mar 18, 2013 & 2452501.200 & 0.349 & 1380 \\
\hline 4. & HN Cas & 2.6594 & Nov 12, 2013 & 2456619.306 & 0.147 & 1200 \\
\hline 5. & V0380 Cas & 1.3577 & Nov 20, 2013 & 255479.656 & 0.075 & 600 \\
\hline 6. & AV Cep & 2.9584 & Oct 13, 2013 & 255643.5478 & 0.020 & 720 \\
\hline 7. & $\mathrm{XY}$ Cet & 2.7807 & Nov 20, 2013 & 2456617.251 & 0.018 & 360 \\
\hline \multirow[t]{2}{*}{8.} & RS CMi & 5.0278 & Feb 20, 2013 & 2448659.708 & 0.412 & 1200 \\
\hline & & & Feb 20, 2014 & 2448659.708 & 0.004 & 1200 \\
\hline 9. & RS CVn & 4.7979 & Apr 23, 2014 & 2448230.537 & 0.101 & 1320 \\
\hline 10. & RR Dra & 2.8313 & Apr 23, 2014 & 2454200.534 & 0.021 & 1320 \\
\hline 11. & TZ Eri & 2.6061 & Nov 20, 2013 & 255478.764 & 0.972 & 720 \\
\hline 12. & AN Gem & 2.0325 & Nov 12, 2013 & 2448394.577 & 0.911 & 1200 \\
\hline \multirow[t]{2}{*}{13.} & SX Gem & 1.3669 & Feb 20, 2013 & 2448656.914 & 0.050 & 1200 \\
\hline & & & Feb 20, 2014 & 2448656.914 & 0.065 & 480 \\
\hline 14. & TW Lac & 3.0374 & Dec 21, 2013 & 2456647.435 & 0.293 & 1200 \\
\hline 15. & FG Lyr & 2.8718 & Apr 23, 2014 & 2456770.916 & 0.028 & 900 \\
\hline \multirow[t]{2}{*}{16.} & BZ Mon & 3.4518 & Feb 20, 2013 & 2448660.263 & 0.070 & 900 \\
\hline & & & Feb 20, 2014 & 2448660.263 & 0.807 & 900 \\
\hline 17. & CH Mon & 6.9223 & Nov 12, 2013 & 2456619.442 & 0.014 & 1200 \\
\hline 18. & FW Mon & 3.8736 & Mar 18, 2013 & 2448630.672 & 0.003 & 1200 \\
\hline \multirow[t]{2}{*}{19.} & HP Mon & 1.4547 & Feb 20, 2013 & 2456344.174 & 0.342 & 1200 \\
\hline & & & Feb 20, 2014 & 2456344.174 & 0.261 & 1200 \\
\hline 20. & RV Oph & 1.3577 & Nov 20, 2013 & 2448634.730 & 0.324 & 1200 \\
\hline 21. & FH Ori & 2.1512 & Oct 14, 2013 & 255581.721 & 0.249 & 900 \\
\hline 22. & Z Ori & 5.2033 & Mar 21, 2014 & 254516.431 & 0.991 & 900 \\
\hline 23. & V0640 Ori & 2.0207 & Oct 13, 2013 & 253008.704 & 0.013 & 1200 \\
\hline \multirow[t]{2}{*}{24.} & CK Per & 2.3728 & Oct 13, 2013 & 2456617.245 & 0.995 & 1800 \\
\hline & & & Nov 20, 2013 & 2456617.245 & 0.015 & 1200 \\
\hline \multirow[t]{3}{*}{25.} & Z Per & 3.0563 & Feb 20, 2013 & 2456343.109 & 0.015 & 720 \\
\hline & & & Oct 13, 2013 & 2456343.109 & 0.016 & 900 \\
\hline & & & Feb 20, 2014 & 2456343.109 & 0.443 & 600 \\
\hline 26. & XY Pup & 14.7783 & Feb 20, 2013 & 2456709.207 & 0.196 & 720 \\
\hline 27. & AC Tau & 2.0434 & Dec 21, 2013 & 2456648.145 & 0.938 & 1200 \\
\hline 28. & RW Tau & 2.7688 & Oct 16, 2013 & 2456582.117 & 0.115 & 480 \\
\hline \multirow[t]{2}{*}{29.} & AF UMa & 5.2576 & Mar 18, 2013 & 2448631.425 & 0.942 & 1200 \\
\hline & & & Mar 21, 2014 & 2448631.425 & 0.940 & 1200 \\
\hline 30. & VV Vul & 3.4114 & Nov 20, 2013 & 2454410.390 & 0.868 & 720 \\
\hline
\end{tabular}


Table 2 The equivalent widths of the spectral lines obtained for the Algols that were observed with HCT

\begin{tabular}{|c|c|c|c|c|c|c|c|c|c|c|}
\hline S.No. & Algol Name & Phase & $\begin{array}{c}\mathrm{H} \delta \\
(4101.70 \AA)\end{array}$ & $\begin{array}{c}\mathrm{H} \gamma \\
(4340.47 \AA)\end{array}$ & $\begin{array}{c}\mathrm{H} \beta \\
(4861.33 \AA)\end{array}$ & $\begin{array}{c}\mathrm{H} \alpha \\
(6562.80 \AA)\end{array}$ & $\begin{array}{c}\mathrm{NaI} \\
(5889.95 \AA+ \\
5895.92 \AA)\end{array}$ & $\begin{array}{c}\text { Ca II } \\
(8498.03 \AA)\end{array}$ & $\begin{array}{c}\text { Ca II } \\
(8542.09 \AA)\end{array}$ & $\begin{array}{r}\text { Ca triplet } \\
(8662.14 \AA)\end{array}$ \\
\hline 1. & V0616 Aql & 0.878 & 10.970 & 7.162 & 3.988 & 3.761 & 1.916 & 0.261 & 1.018 & 1.339 \\
\hline 2. & V0769 Aql & 0.977 & 2.541 & 3.876 & 4.664 & 3.411 & 0.968 & 1.095 & 1.885 & 2.591 \\
\hline 3. & V1340 Aql & 0.349 & 0.284 & 5.909 & 3.124 & 3.067 & 0.428 & 0.167 & - & 0.136 \\
\hline 4. & HN Cas & 0.147 & 12.110 & 9.818 & 8.806 & 2.638 & 1.935 & 0.618 & 0.263 & 0.843 \\
\hline 5. & V0380 Cas & 0.706 & 11.820 & 11.730 & 13.460 & 7.816 & 1.108 & 0.910 & 1.655 & 5.379 \\
\hline 6. & AV Cep & 0.384 & 8.494 & 10.600 & 7.414 & 1.475 & 1.636 & 1.598 & 0.941 & 10.130 \\
\hline 7. & XY Cet & 0.018 & 6.202 & 4.409 & 7.159 & 6.144 & 0.301 & 1.003 & 1.227 & 1.953 \\
\hline 8. & RS CMi & 0.412 & 11.010 & 13.370 & 5.012 & 4.095 & 1.256 & 0.765 & 0.080 & 0.393 \\
\hline 9. & RS CVn & 0.101 & 2.877 & 1.744 & 2.745 & 1.043 & 1.354 & 0.991 & 1.973 & 2.060 \\
\hline 10. & RR Dra & 0.021 & 11.060 & 10.500 & 12.360 & 0.974 & 1.398 & 1.486 & 2.241 & 1.807 \\
\hline 11. & TZ Eri & 0.071 & 6.888 & 4.737 & 7.622 & 3.716 & 1.766 & 1.913 & 3.642 & 3.362 \\
\hline 12. & AN Gem & 0.911 & 4.346 & 19.600 & 7.176 & 5.424 & 0.504 & 0.216 & 1.208 & 9.990 \\
\hline \multirow[t]{2}{*}{13.} & SX Gem & 0.050 & 9.676 & 10.140 & 8.992 & 5.754 & 1.345 & 0.591 & 1.094 & 1.201 \\
\hline & & 0.065 & 10.200 & 10.410 & 8.790 & 6.112 & 1.322 & 0.501 & 1.146 & 2.525 \\
\hline 14. & TW Lac & 0.293 & 8.559 & 9.155 & 7.726 & 5.362 & 1.322 & 0.501 & 1.146 & 2.525 \\
\hline 15. & FG Lyr & 0.028 & 2.437 & 2.283 & 2.616 & 1.887 & 2.772 & 0.946 & 2.192 & 1.071 \\
\hline \multirow[t]{2}{*}{16.} & BZ Mon & 0.070 & 0.663 & 4.252 & 2.315 & 1.263 & 0.117 & - & - & - \\
\hline & & 0.806 & 13.010 & 9.118 & 6.619 & 0.111 & 0.071 & - & - & - \\
\hline 17. & CH Mon & 0.014 & 8.302 & 8.190 & 5.493 & 1.029 & 2.051 & 0.731 & 2.008 & 0.328 \\
\hline 18. & FW Mon & 0.022 & 16.680 & 4.690 & 4.228 & 2.196 & 1.296 & 0.247 & 0.346 & 3.520 \\
\hline \multirow[t]{2}{*}{19.} & HP Mon & 0.342 & 1.471 & 14.820 & 3.136 & 5.877 & 1.628 & 0.086 & 1.076 & 0.659 \\
\hline & & 0.260 & 6.594 & 7.184 & 12.250 & 6.640 & 0.992 & 0.298 & 1.123 & 0.694 \\
\hline 20. & RV Oph & 0.324 & 11.620 & 11.130 & 9.907 & 6.107 & 1.354 & 0.991 & 1.973 & 2.060 \\
\hline 21. & FH Ori & 0.249 & 11.350 & 5.984 & 3.323 & 7.141 & 1.405 & 1.077 & 9.734 & 3.037 \\
\hline 22. & Z Ori & 0.991 & 4.714 & 5.648 & 4.773 & 3.511 & 0.437 & 0.479 & 1.723 & 0.646 \\
\hline 23. & V0640 Ori & 0.013 & 2.546 & 4.816 & 5.433 & 4.061 & 1.445 & 1.195 & 1.577 & 1.719 \\
\hline 24. & CK Per & 0.995 & 1.100 & 2.762 & 3.517 & 2.105 & 0.556 & 0.304 & 0.429 & 0.323 \\
\hline \multirow[t]{3}{*}{25.} & Z Per & 0.015 & 11.650 & 11.420 & 10.950 & 6.736 & 0.515 & 0.925 & 1.879 & 3.797 \\
\hline & & 0.016 & 11.300 & 11.840 & 11.580 & 7.032 & 1.190 & 0.355 & 2.105 & 1.953 \\
\hline & & 0.443 & 11.640 & 11.970 & 12.650 & 7.358 & 0.979 & 0.953 & 2.260 & 2.295 \\
\hline 26. & $\mathrm{XY}$ Pun ${ }^{2}$ & 0.195 & -4.605 & 0.744 & -1.891 & $-3,585$ & 3.872 & 1.316 & 2.208 & 1.655 \\
\hline \multirow[t]{2}{*}{27.} & AC Tau & 0.278 & 6.723 & 7.445 & 5.691 & 4.464 & 0.323 & 2.920 & 1.360 & 0.995 \\
\hline & & 0.938 & 3.571 & 5.073 & 5.391 & 4.285 & 0.396 & 0.449 & 1.652 & 3.397 \\
\hline 28. & RW Tau & 0.116 & 9.082 & 9.684 & 7.869 & 5.805 & 1.333 & 0.311 & 1.068 & 1.187 \\
\hline \multirow[t]{2}{*}{29.} & AF UMa & 0.942 & 9.127 & 12.370 & 12.470 & 2.092 & 0.101 & 0.577 & 0.209 & 6.369 \\
\hline & & 0.940 & 13.050 & 15.850 & 14.300 & 8.766 & 0.358 & 6.112 & 0.760 & 9.880 \\
\hline 30. & VV Vul & 0.869 & 11.070 & 10.560 & 9.785 & 6.715 & 1.644 & 0.084 & 2.097 & 2.417 \\
\hline
\end{tabular}




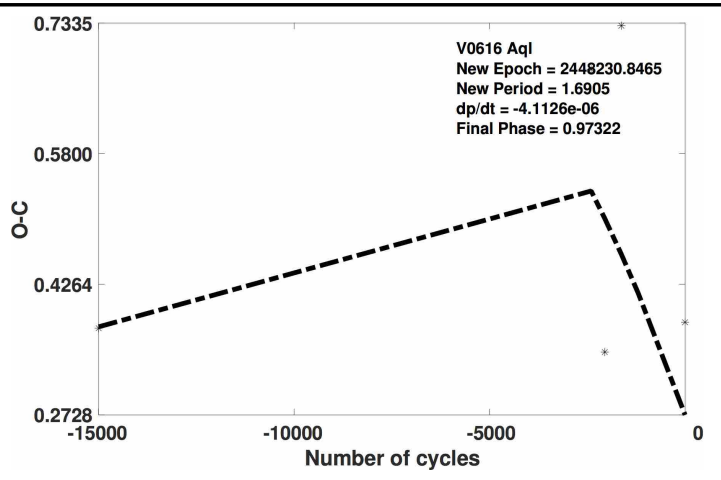

Fig. 2 O-C diagram of V0616 Aql.

One spectra for V0616 Aql was obtained on Apr 23, 2014 and the phase of observation calculated from the derived epoch is 0.9732. Spectral lines and their dominant profiles obtained can be seen in Figure 1. All the Balmer lines in the selected wavelength range show good absorption profiles with equivalent widths as shown in Table 2. It is observed that $\mathrm{H} \alpha$ and $\mathrm{H} \beta$ lines show a greater fill in the absorption profiles than other Balmer lines.

\subsection{V0769 Aql}

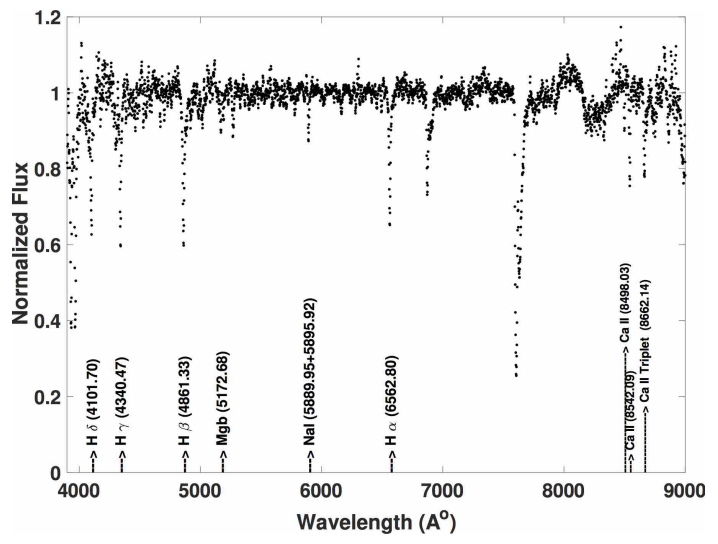

Fig. 3 Spectrum of V0769 Aql.

V0769 Aql (= GSC 05164-01535, V=15.40) was catalogued as an Algol type close binary by Budding et al. (2004) and Malkov et al. (2006). This is least studied Algol binary with period derived as $4^{d} .5623$ Kukarkin et al. (1971). The spectral type derived to be as G7V using the 2MASS magnitudes by Cutri et al. (2003). Due to lack of latest data, the available epoch in the literature was taken to calculate the phase. No period study was done due to unavailability of data. One spectrum for the variable was obtained on Nov 20, 2013 at phase 0.9773 . The dominant Balmer line profiles in the spectrum are presented for the first time in this paper (Figure 3 and the equivalent widths obtained are given in Table 2 Due to the observed phase lying close to primary minima, the obtained spectra can be representing the cooler evolved secondary and its environment. However, the best fit spectral model couldn't be obtained using Jacoby spectral library.

\section{$3.3 \mathrm{~V} 1340 \mathrm{Aql}$}

V1340 Aql(=2MASS J18441601-0330147, $\mathrm{B}=14.3$ ) is also one of the least studied Algol with a period of $1^{d} .596940$ (Avvakumova et al. (2013). It was identified as a variable star by Kurochkin (1954); Kholopov et al. (1981) and later was catalogued as Algol type by Budding et al. (2004) and Malkov et al. (2006). No period study was done on this variable due to lack of data. One spectrum for V1340 Aql at phase 0.3485 as calculated from available epoch, was obtained on Mar 18, 2013. We report the dominant Balmer lines in the spectrum for the first time. The spectrum is shown in Figure 4 and corresponding equivalent widths are given in Table 2. All the spectral lines are in absorption. $\mathrm{H} \delta$ shows a weaker absorption compared to other Balmer lines.

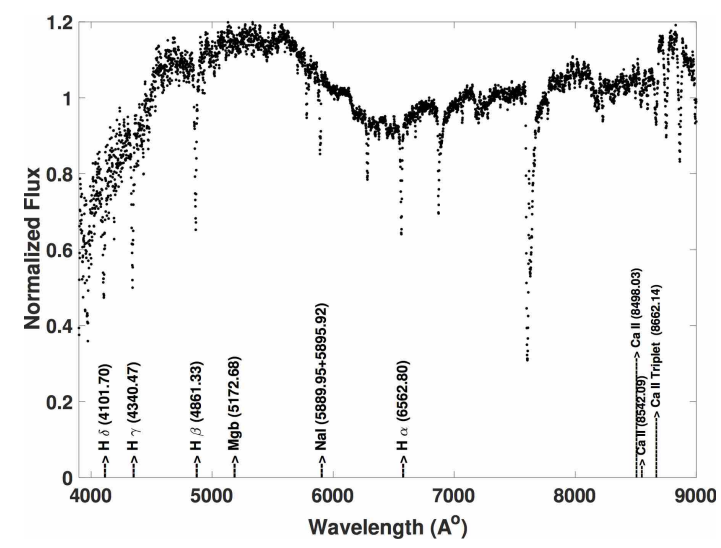

Fig. 4 Spectrum of V1340 Aql.

\subsection{HN Cas}

HN Cas (GSC 03672-01509, 2MASS J01005435 + 5554178, $\mathrm{V}=15.45$ ) was classified as a variable star by Hoffmeister (1943); Ahnert et al. (1947a) and the period of the variable is $2^{d} .6594$ (Kukarkin et al. (1971)). Later it was catalogued as Algol by Kinnunen and Skiff (2000), Budding et al. (2004) and Malkov et al. (2006). Few times of minima (ToM) were given by Brát et al. (2007) which is insufficient to carry out period study. Further no detailed study was carried out for the variable so far. One spectra for HN Cas at phase 0.1466 (as calculated from the available epoch) was obtained on Nov 12, 2013. The dominant Balmer spectral lines can be seen in Figure 5 and equivalent widths are given in Table 2. It is observed that $\mathrm{H} \alpha$ absorption profile is relatively less prominent than the other 
Balmer lines which could be due to fill in effect. The spectral class determined in the current study using best fit spectral model (Jacoby et al. (1984)) is Al V.

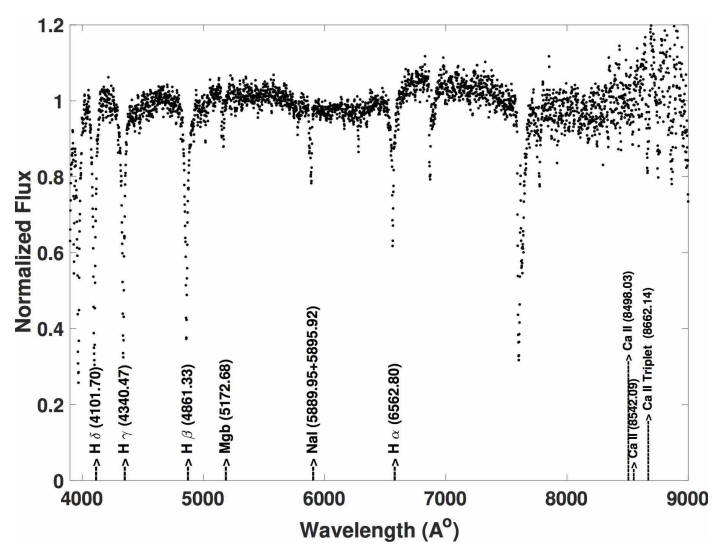

Fig. 5 Spectrum of HN Cas.

\subsection{V0380 Cas}

V0380 Cas (=GSC 04307-01121= TYC 4307-1121-1) was first discovered by Bauernfeind in 1899 and its eclipsing nature was studied by Strohmeier and Bauernfeind (1968). Meinunger (1965) has classified its spectral type as $\mathrm{A} 0$ and orbital elements were first given by Brancewicz and Dworak (1980). This is a well studied Algol binary with current period given as $1^{d} .357270$ by Christopoulou et al. (2011) . They carried out first photometric study and found it to be a well-detached system with moderately evolved main-sequence components and a high resolution spectroscopy is recommended for this system to determine its evolutionary status. They also concluded from the period study that there is no indication of period change, eliminating the possibility of third companion. Hence no period period study was done in the current work. One spectrum for V0380 Cas was obtained on Nov 20, 2013 at phase 0.0749. The dominant Balmer line profiles can be seen in Figure 6 and the derived equivalent widths are given in Table 2

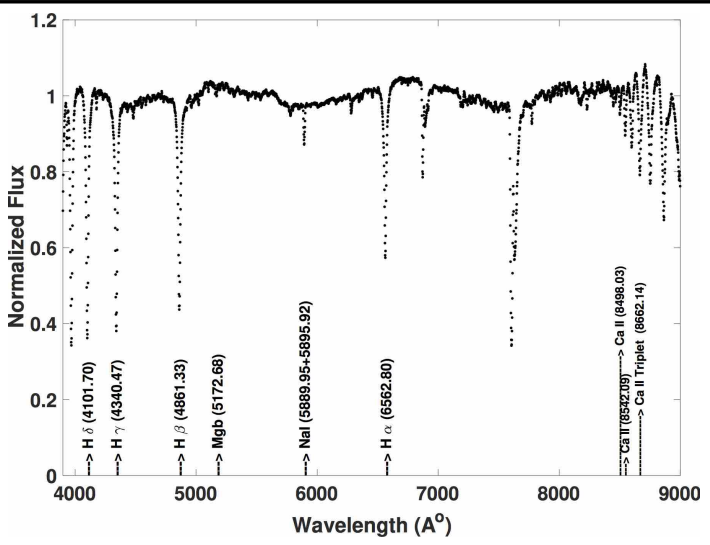

Fig. 6 Spectrum of V0380 Cas.

3.6 AV Cep

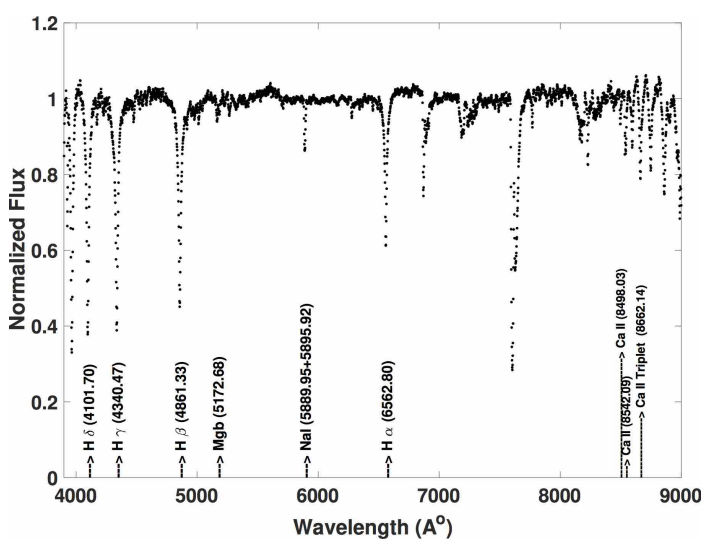

Fig. 7 Spectrum of AV Cep.

AV Cep (= 2MASS J05542644+8601206,V=12.18) was first identified and catalogued as eclipsing binary by Kukarkin et al. (1971). It was later classified as an Algol type by Budding et al. (2004) and the updated period of the variable was given as $2^{d} .958100$ (Malkov et al. (2006)). Photoelectric minima (Agerer and Hubscher [2003); Hubscher (2007); Hubscher et al. (2012)) and few ToM (Borovicka (1993); Kreiner (2004)) are available in the literature. However, the ToM in the literature are very few to carry out O-C studies. One spectrum for AV Cep was obtained on Oct 13, 2013 at phase 0.0199 (calculated from the latest epoch available in the literature). Dominant spectral lines are shown in Figure 7 and the calculated equivalent widths are given in Table 2 From the observed phase the spectra can be attributed to the secondary component and it is also observed that $\mathrm{H} \alpha$ absorption profile is relatively less prominent than the other Balmer lines which could be due to fill in effect.The spectral class determined in this current study using best fit spectral model (Jacoby et al. (1984)) is F5 II. 


\section{$3.7 \mathrm{XY}$ Cet}

$\mathrm{XY}$ Cet(=TYC 51-832-1, V=8.75) is a well studied Algol type binary. It was first discovered by Strohmeier and Knigge (1961) and was photoelectrically observed by Morrison and Morrison (1968). Detailed analysis of this binary was extensively done by many authors. The current period as derived by Smalley et al. 2014) is $2^{d} .780710$. Period studies were carried out by many authors beginning with Srivastava (1988) to Southworth et al. 2011) however, no period variations were reported so far. Hence no period study was done in the present work. Popper (1971) obtained first spectroscopic data of this Algol and proposed its spectral type as A2 and F0 by inspecting metallic lines. This was further validated and refined using photometry data from superWASP study (Southworth et al. (2011)). Eker et al. 2014 2015) have deduced the double-lined binary nature and spectral type by disentangling previously observed data for the variable. In the current work one spectrum for XY Cet was obtained on Nov 20, 2013. From the latest epoch available the phase calculated is 0.0179 . The dominant profiles in the spectra are shown in Figure 8 and the equivalent widths calculated are given in Table 2. The spectral type determined using (Jacoby et al. (1984) is F0 III which is in agreement with that deduced from the work done by Eker et al. (2015).

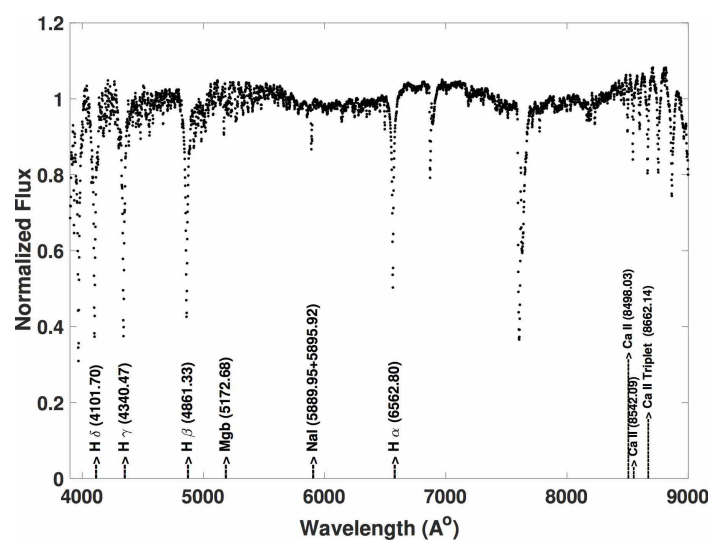

Fig. 8 Spectrum of XY Cet.

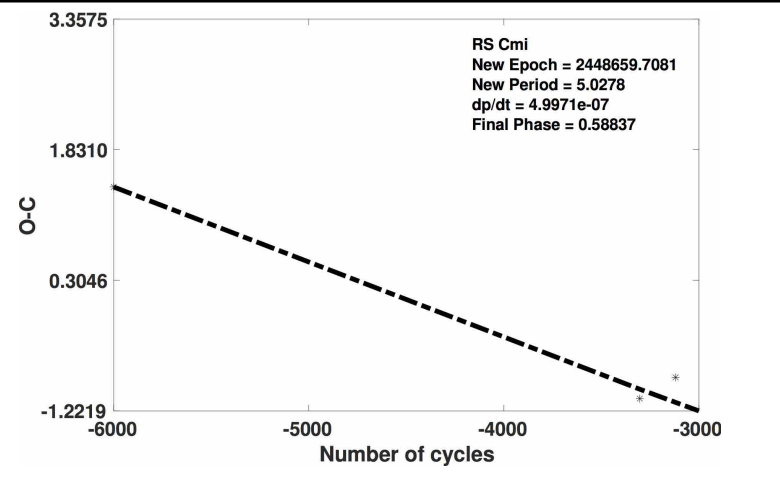

Fig. 9 O-C diagram of RS CMi.

\subsection{RS CMi}

RS CMi (=AN 154.1928, $\mathrm{B}=13.8$ ) is an Algol-type eclipsing binary with an orbital period of $5^{d} .027800$ (Avvakumova et al. (2013)). It was discovered by Hoffmeister (1928). Budding et al. (2004) and Malkov et al. (2006) have presented this variable in the catalogue of Algol type binary stars. Many authors Diethelm (2005); Hubscher (2007); Hubscher et al. (2009) have presented ToM for this binary. It is one of the least studied Algols with only 5 ToM available of which a group of 4 are spread around $7 \mathrm{yrs}$ and one observation separated from the group by about 60 years. We present the first period and spectroscopic study. The best fit for the O-C plot in Figure 9 shows only a decreasing trend of period at the rate of $\mathrm{dp} / \mathrm{dt}=$ $4.9971 \times 10^{-7}$ days/year. The new epoch obtained from current study is HJD (Min I) $=2448659.708+5^{d} .027755$ $\times$ E. Two spectra were obtained for RS CMi on Feb 20, 2013 and on Feb 20, 2014 at phases 0.4117 \& 0.0044, respectively calculated from the new epoch. The spectral lines identified are shown in Figure 10 and the equivalent widths of the dominant lines are given in Table 2 . From the spectral study it is observed that the absorption profile for the $\mathrm{H} \alpha$ line is relatively less prominent when compared to other Balmer lines indicating fill in effect for the primary component. At the phase outside eclipse both $\mathrm{H} \alpha$ \& $\mathrm{H} \beta$ show fill in effect in the absorption profile relative to other Balmer lines. This can be related to the evolution of disk around the current primary, which can be confirmed with further observations. Based on mini- 
mum res ${ }^{2}$ and visual inspections, the spectral class is derived to be A3 III (Jacoby et al. (1984)).

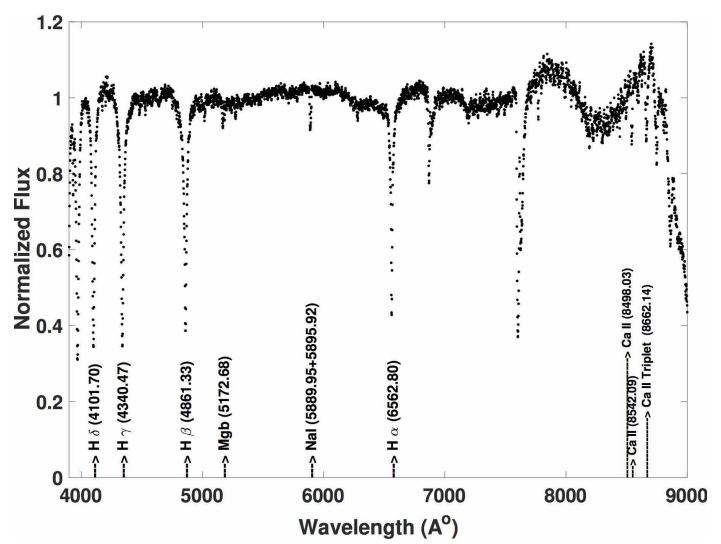

Fig. 10a Spectrum of RS CMi (2013).

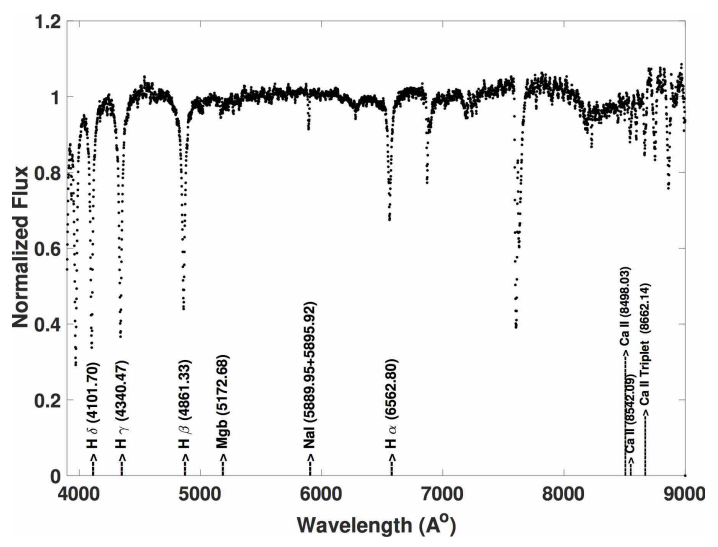

Fig. 10b Spectrum of RS CMi (2014).

\subsection{RS CVn}

RS CVn (= GSC 02534-01642 = AN 10.1914, V=7.93) is one of the most interesting and well studied Algol type binary since its discovery by Hoffmeister (1915) along with 5 times of minima. It has an orbital period of $4^{d} .797900$ (Kukarkin et al. (1971)) and spectral class F6IV+G8IV (Strassmeier and Fekel (1990)). Photometric and spectroscopic investigations revealed the orbital elements and spectral types of the components as F3 \& K0 by Popper (1980). It was observed that there is a cyclic fluctuation in the primary period which was mostly due to mass loss during continuous ejection of particles from migrating active regions like star spots on the cooler star. It was also hypothesized that the variable is in pre-main sequence contraction and the cooler star shows a T-Tauri star characteristics (Sitterly (1921); Joy (1922); Keller and Limber (1951); Plavec and Smetanova (1959); Hall (1972);
Arnold and Hall (1973); Catalano and Rodono (1974); Rhombs and Fix (1976)). In addition, the spectral studies carried out by Fernández-Figueroa et al. (1994) in the region of Ca II $\mathrm{H} \& \mathrm{~K}$ for the variable confirmed emission flux variations with the orbital phases. The ultraviolet excess characterizing RS CVn spectra was attributed to the free free emission from hot circumstellar gas by Rhombs and Fix (1976) . In the current work this is an Algol type with 18 observations of ToM is plotted for the period study. 17 of the observations are grouped around 40 years and this group is separated by one of the observations by about 46 years. However the data shows a sinusoidal component of period variations superimposed on the decreasing trend (Figure 11 ) of the best fit curve. A quadratic ephemeris to fit the $\mathrm{O}-\mathrm{C}$ variation is derived to be $\mathrm{dp} / \mathrm{dt}=-2.9951 \times 10^{-6}$ days/year. The new epoch deduced is HJD $($ Min I $)=2448230.537+4^{d} .797852 \times \mathrm{E}$. Mass exchange or mass loss or presence of third body can be excluded (Lanza and Rodonò (2004); Frasca and Lanza (2005) for variables showing magnetic activity. The magnetic activity is evident in RS CVn (FernándezFigueroa et al. (1994)) attributing to the sinusoidal period changes. One spectrum for RS CVn was obtained on Apr 23, 2014 at phase 0.1010 calculated from the derived epoch. No spectroscopy work was carried out for the past three decades. In the current study we present the spectrum with dominant spectral lines observed as shown in Figure 13 and the equivalent widths calculated in Table 2 the light curve obtained using data available in the literature is shown in Figure 12 . The Balmer lines in the spectra show weak absorption profiles which could be due to fill in effect caused by activity on or near the primary component.

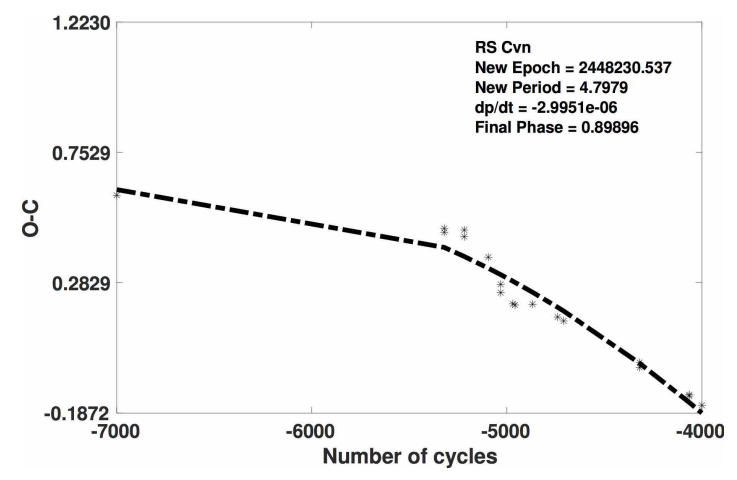

Fig. 11 O-C diagram of RS CVn. 


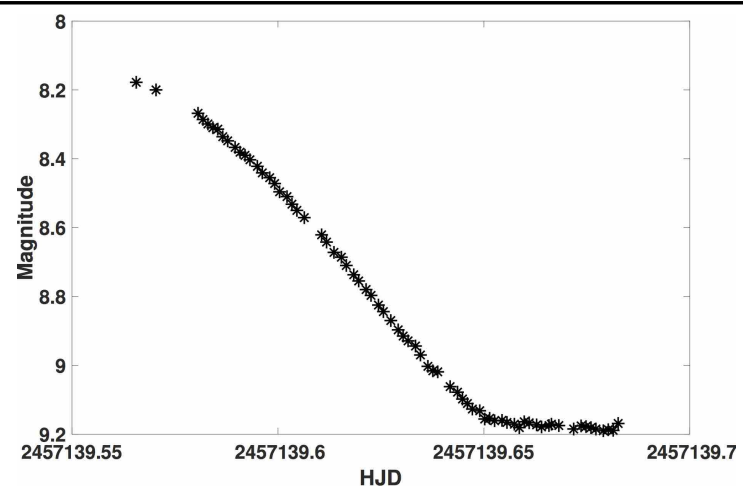

Fig. 12 Light curve of RS CVn.

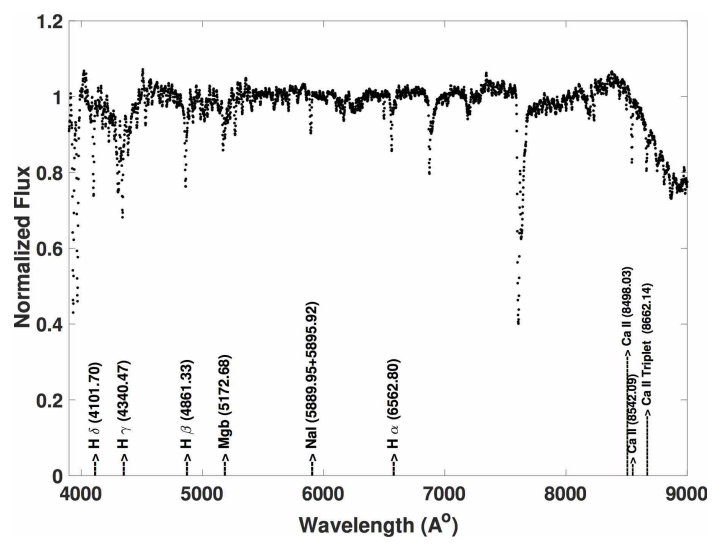

Fig. 13 Spectrum of RS CVn.

\subsection{RR Dra}

RR Dra (=AN 188.1904, V=9.831) is an Algol-type eclipsing binary with an orbital period of $2^{d} .831200$ (Kukarkin et al. (1971)). It contains an A2 type primary and a K0type secondary (Yoon et al. (1994)). Many times of light minimum of RR Dra were collected from previous work (Dugan and Wright (1939); Szczepanowska (1956 1959); Whitney (1957)) and extensive period study was carried out for this variable showing a secular change with rapid period increase rate of $\mathrm{dp} / \mathrm{dt}=+4.24 \mathrm{E} 10^{-6}$ days/year Qian et al. (2002)). A spectrum was obtained at phase 0.0210 (calculated using latest epoch available in the literature) on April 23, 2014. The dominant spectral lines identified are shown in Figure 15 their equivalent widths are given in Table 2 and the light curve is shown in Figure 14 The equivalent width of the $\mathrm{H} \alpha$ line shows a remarkably high fill in effect compared to other Balmer lines of all the Algols in current study. The work done by Qian et al. (2002) suggests that the rapid period changes superimposed on the long-term increases could be due to the structural variation of the cool mass-loser through the instabilities in the convective outer layer (COL) or through cyclic magnetic activity of the K0-type components. Since for this variable the period jumps are not observed in a short-term alternating way, the most possible explanation for period changes could be the structural change caused by instabilities in the COL via a dynamical mass loss from the cool subgiant. When the parameters were plotted (Figure 52 on the available models (Lubow and Shu (1975); Kaitchuck (1985)), the position of RR Dra is observed to be above the $\omega_{\text {dis }}$ implying that it is similar to those systems with permanent double peaked emission lines that may vary in strength and shape. It also indicates that stable disk could not form but circumstellar bulges may be possible, thus validating Qian's study.

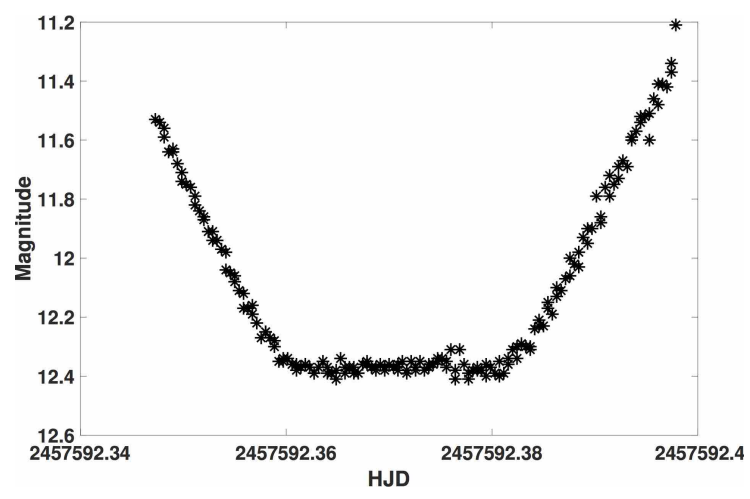

Fig. 14 Light curve of RR Dra.

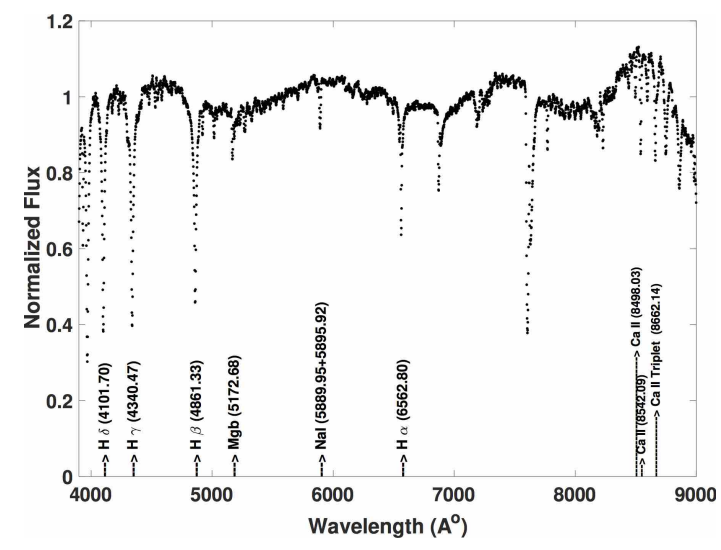

Fig. 15 Spectrum of RRDra.

\subsection{TZ Eri}

TZ Eri (= GSC 04147-01115 = TYC 4147-1115-1, V= 10.60) is an Algol-type eclipsing binary with an orbital period $2^{d} .6061$ (Kreiner (2004)). Its variability was discovered by Hoffmeister (1929) and spectral class was first classified by Cannon (1934) as F type. Many authors have (Kaitchuck and Honeycutt (1982); Kaitchuck and Park 
(1988); Vesper et al. (2001)) have studied the variable and given the parameters predicting the presence of an accretion disc in the system. The variable's possible link between the orbital and pulsational periods was investigated by Soydugan et al. (2006). Observed minima were given by many authors Kordylewski (1963); Mallama (1980); Faulkner and Kaitchuck (1983); Samolyk 2008 2010, 2011) and Harmanec (1988) detected a third body in both light curve solutions as well as spectra. Liakos and Niarchos 2009) has published frequency analysis and the period study reveals a long-term period increase attributed to mass transfer from secondary component to primary component (Zasche et al. (2008)). The spectral types of the components are given as A5/6 for primary and K0/1 III for secondary by Barblan et al.(1998) and its pulsational behaviour was detected by Mkrtichian et al. (2005).

A spectrum of TZ Eri was obtained at phase 0.071 on Nov 20, 2013. The dominant spectral lines identified are given in Figure 17. The light curve is shown in Figure 16 and the equivalent widths in Table 2 The $\mathrm{H} \alpha$ absorption profile is observed to be relatively less prominent than the other Balmer lines. The new phase obtained using derived ephemeris is 0.97171 . From the available models Lubow and Shu (1975) and Kaitchuck (1985), the position of TZ Eri is below the $\omega_{\text {min }}$ line showing primary radius larger than $\omega_{d i s k}$. This implies that a stable accretion disk could not form but circumstellar bulges may prevail.

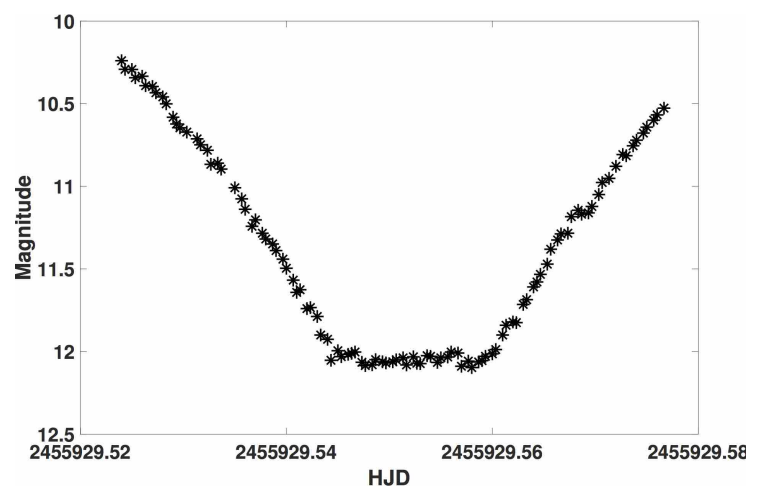

Fig. 16 Light curve of TZ Eri.

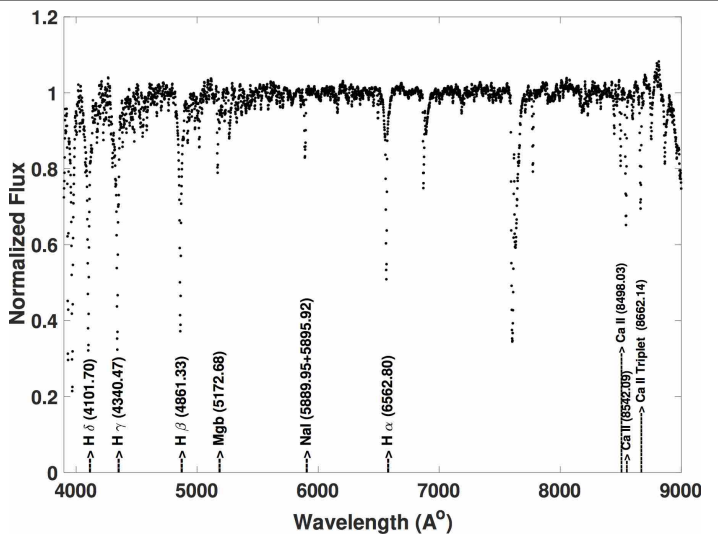

Fig. 17 Spectrum of TZ Eri.

\subsection{AN Gem}

AN Gem (2MASS J07085752+1948136, $\mathrm{V}=13.20$ ) is an Algol-type eclipsing binary with an orbital period $2^{d}$ .032300 (Kreiner (2004)). The linear elements for this variable were given by authors Borovicka (1993) Hubscher (2005) \& Kreiner (2004). No further work was carried out on this variable so far. First period study is presented in the current work and from the quadratic ephemeris the $\mathrm{O}-\mathrm{C}$ variation is calculated. The $\mathrm{O}-\mathrm{C}$ diagram is shown in Figure 18 has been plotted for only 3 data points spanning over 11 years and one point separated by 66 years. The fit suggests a decreasing period. The decreasing rate of period obtained is $\mathrm{dp} / \mathrm{dt}$ $=-2.4932 \times 10^{-6}$ days/year, however additional observations are required to understand the change clearly. The new ephemeris obtained is as follows HJD (Min I) = $2448394.577+2^{d} .03246 \times$ E. A spectrum for AN Gem was obtained on Nov 12, 2013 at phase 0.911 and the spectral lines identified are shown in Figure 19 and the equivalent widths in Table2, Based on minimum res ${ }^{2}$ and visual inspections, the best fit spectral model (Jacoby et al. (1984)) was selected to determine the spectral class as F3 III.

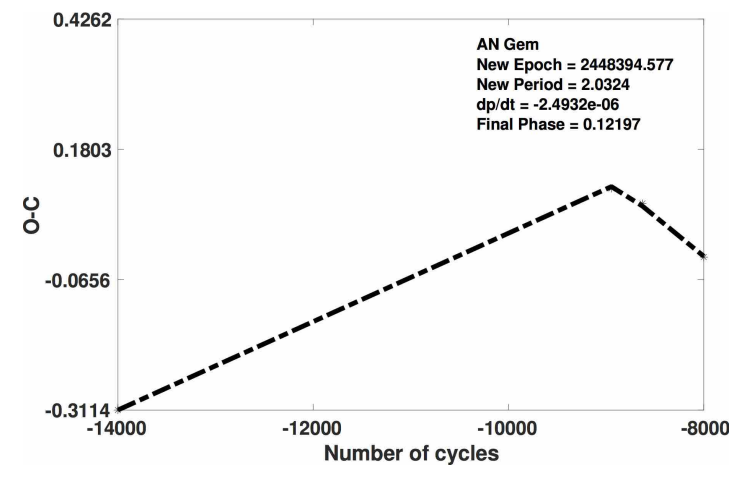

Fig. 18 O-C diagram of AN Gem. 
11

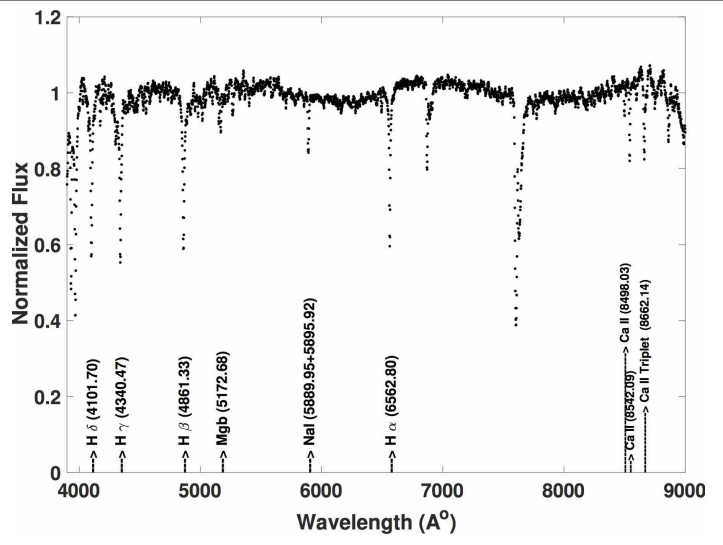

Fig. 19 Spectrum of AN Gem.

\subsection{SX Gem}

SX Gem (=AN 56.1908, V=11.00) is an Algol-type eclipsing binary with an orbital period $1^{d} .366900$ with spectral class A0+A9 (Giuricin et al. (1984)). It was first listed in the catalogue of variable stars by Gaposchkin (1932). Absolute parameters for this variable were deduced by Brancewicz and Dworak (1980). Many authors have recorded ToM but no period variation studies were done. Zasche (2011) has derived basic parameters for SX Gem and it is observed that the mass ratio is different in his study when compared to that derived by Brancewicz and Dworak (1980).

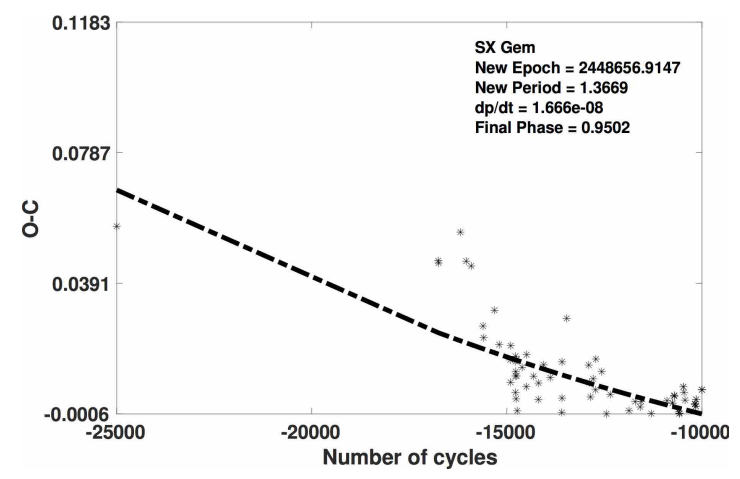

Fig. 20 O-C diagram of SX Gem.

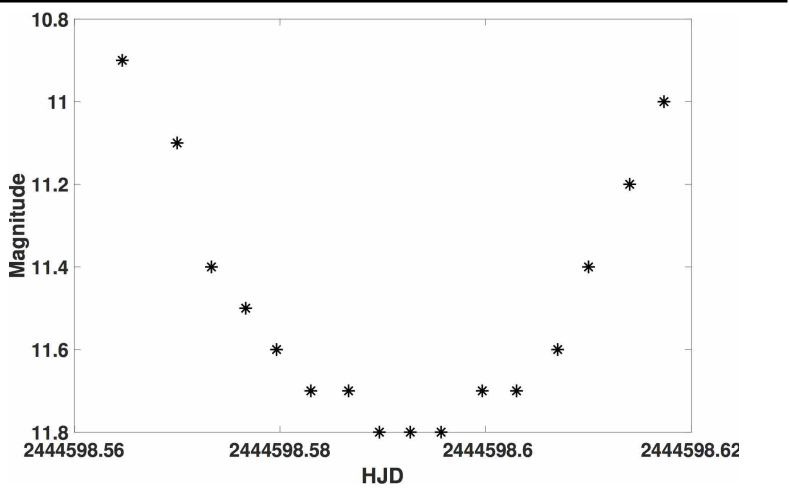

Fig. 21 Light curve of SX Gem.

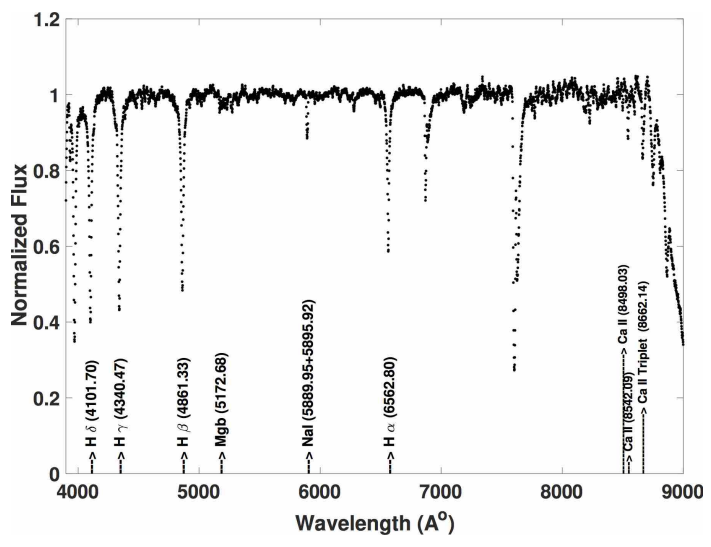

Fig. 22a Spectrum of SX Gem (2013).

The (O-C) is plotted (Figure 20) for 86 observations of ToM with a group of 85 data points scattered around 45 years and separated from one observation by another 45 years. The observations show a large scatter around the best fit curve, which is clearly showing a decreasing trend of the period and the rate of period decrease obtained is $\mathrm{dp} / \mathrm{dt}=1.666 \times 10^{-8}$ days/year. The decreasing trend of the period is in agreement with the change in mass ratio of the variable. The new ephemeris obtained is as follows HJD $($ Min I $)=2448656.9147+1^{d} .366877 \times$ E. The light curve obtained from the data available in the literature is shown in Figure 21. Two spectra for SX Gem were observed on Feb 20, 2013 and Feb 20, 2014 at phases 0.050 and 0.065 , obtained using latest epoch in the literature, respectively as shown in Figure 22 


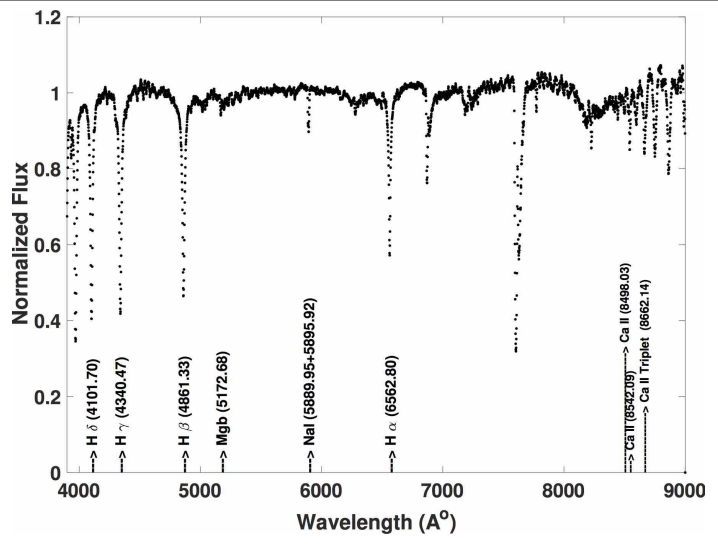

Fig. 22b Spectrum of SX Gem (2014).

\subsection{TW Lac}

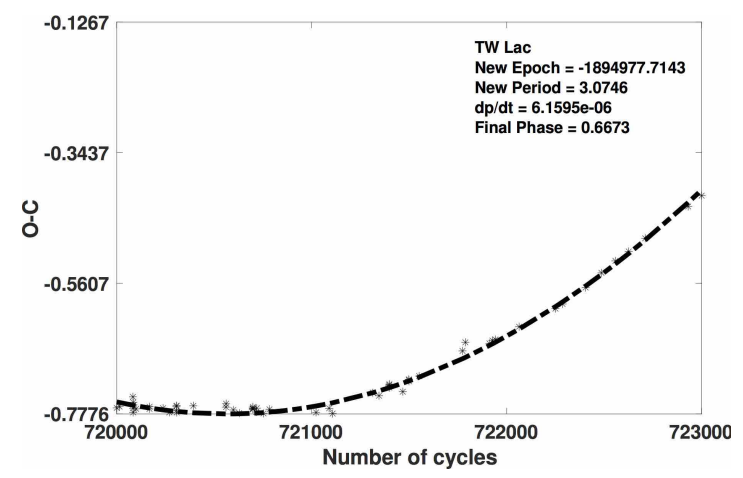

Fig. 23 O-C diagram of TW Lac.

TW Lac (=GSC 03987-01714=AN $123.1925, \mathrm{~V}=11.85$ ) is a detached Algol type binary. Its variability was first studied by Gaposchkin (1932), which led to extensive observations of minima for this variable. The spectral type was derived to be A3 IV by Halbedel (1984) and period $3^{d} .037518$ Kreiner (2004)). It was catalogued as Algoltype by Budding et al. (2004) and was categorized as a candidate semi detached system for pulsation by Soydugan et al. (2006). The minima and period changes were intensively studied by many authors Whitney 1957 1959); Wood and Forbes (1963); Kreiner (1971); Agerer and Hubscher (2003); Malkov et al. (2006); Erdem et al. 2007); Dogru et al. (2007). In the current study the period study has been performed on 65 observations of ToM. The data shows a distinct sinusoidally varying period as shown in the Figure 23 it is also observed that there is a sinusoidal component superimposed on increasing period $\mathrm{O}-\mathrm{C}$ curve.The epoch derived is as follows $\mathrm{HJD}(\mathrm{Min} \mathrm{I})=$ $2456647.435+3^{d} .074560 \times \mathrm{E}$ and the variation in period is $\mathrm{dp} / \mathrm{dt}=6.1595 \times 10^{-6}$ days $/$ year.

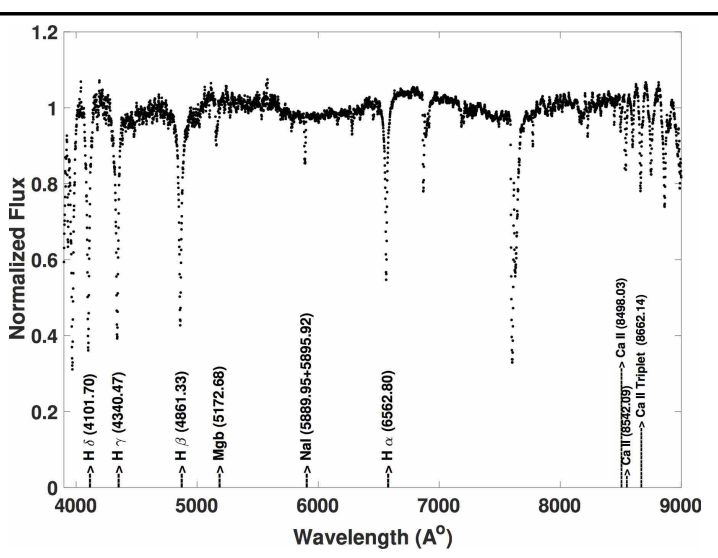

Fig. 24 Spectrum of TW Lac.

Though the first spectral study was done by Halbedel (1984), no information was provided about the spectra except for spectral type. Kaitchuck et al. (1985) have done spectroscopy of 52 short period Algols during their primary eclipse phase, and have tried to model the observed spectral line profiles to understand the nature of the accretion disk around the primary. They have found that when observed in July 1983, during eclipse which is a total eclipse, there is no emission in the observed Balmer line profiles $(\mathrm{H} \beta$ to $\mathrm{H} \gamma)$ and the approximate maximum equivalent width of emission was less than 0.4. Their study indicates that the system is not harboring a transient disc. We have done the spectral study for the spectra obtained on Dec 21, 2013 at phase 0.293 calculated from the latest epoch obtained. The spectrum showing the dominant spectral lines is shown in Figure 24 and their equivalent widths in Table 2 . It is observed that all the Balmer lines show strong absorption profile at the observed phase that is out of eclipse. The spectral type obtained using (Jacoby et al. (1984)) stellar library is A8 V.

\subsection{FG Lyr}

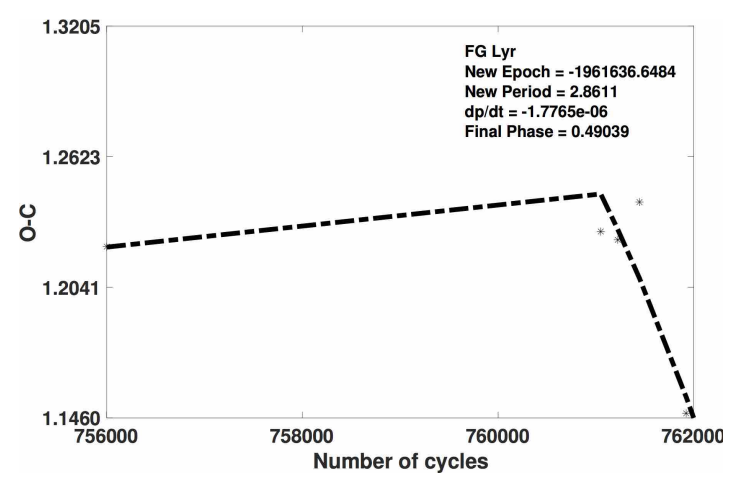

Fig. 25 O-C diagram of FG Lyr. 


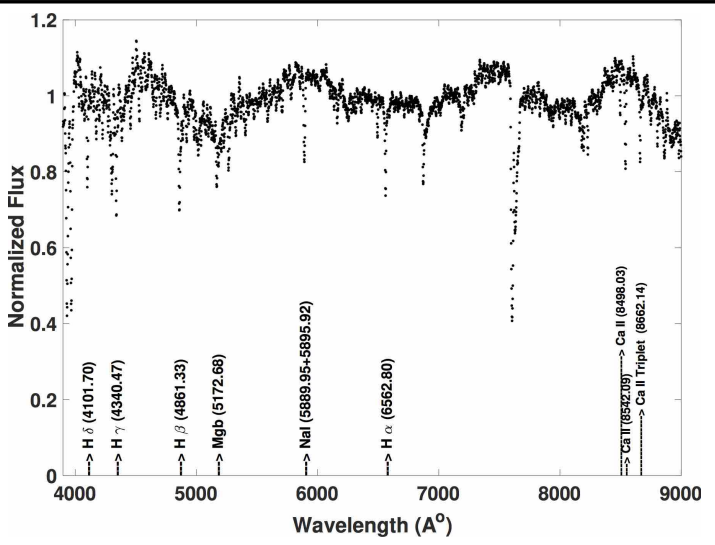

Fig. 26 Spectrum of FG Lyr.

FG Lyr (= AN 45.1930, V = 12.4) is an Algol-type eclipsing binary with an orbital period $2^{d} .87183$ Kreiner (2004). Dworak (1977) published minima from the Cracow observations of variable stars made during the years 19201950. Later its photoelectric minima were determined by Agerer and Hubscher (2001); Hubscher (2005). No detailed study on the orbital period change of the system has been done so far. We present the first period study. There are only six data points with 5 spread over 15 years and one point separated by 78 years in the literature. The best fit on the observed data shows a decreasing period and the $\mathrm{O}-\mathrm{C}$ diagram represents the variation as shown in Figure 25 with the rate of period decrease dp/dt as $1.7765 \times 10^{-6}$ days/year. The new epoch derived from the present study is as follows HJD (Min I) $=2456770.916+$ $2^{d} .86112 \times$ E. A spectrum was obtained on April 23, 2014 for this variable at phase 0.0277 and the spectral lines identified are shown in Figure 26 and equivalent widths are given in Table 2. The best fit spectral model was selected (Jacoby et al. (1984)) to determine the spectral class as G6 III, representing the spectral type of evolved secondary component.

\subsection{BZ Mon}

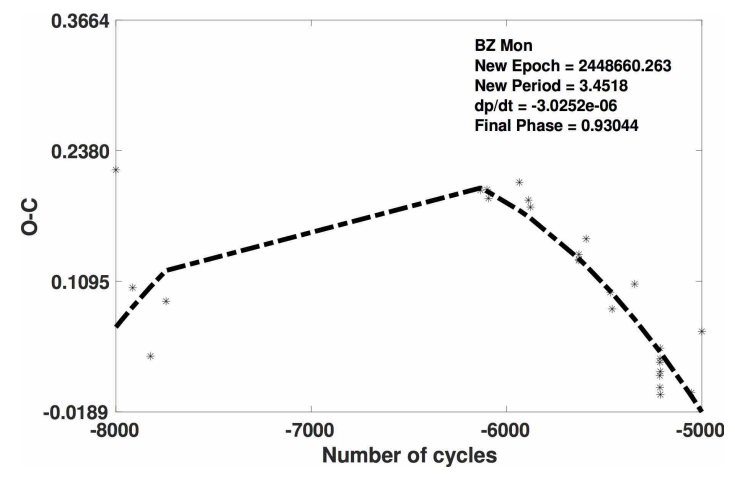

Fig. 27 O-C diagram of BZ Mon.

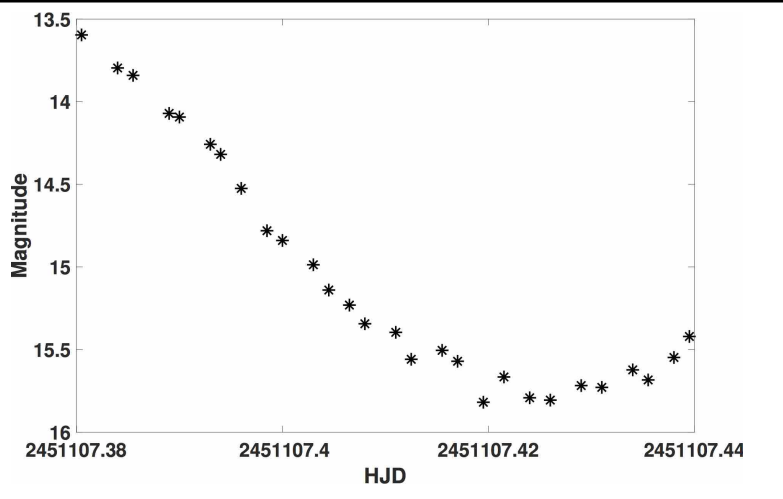

Fig. 28 Light curve of BZ Mon.

BZ Mon(= AN 63.1936, $\mathrm{V}=12.10)$ is an Algol-type eclipsing binary with an orbital period $3^{d} .451721$ (Avvakumova et al. (2013)). The variable has a rich observational history and was first discovered by Hoffmeister (1936) and later basic data on light curve was given by many authors, Ahnert and Hoffmeister (1943); Ahnert et al. (1947a) with a major contribution from Schaefer (1980). He has given an improved ephemeris included in GCVS Min I $=$ HJD2443192.663 $+3^{d} .451804$ Zakirov (2001) has conducted first photoelectric UBVR observations in 1998-99 and has determined new ephemeris, component spectral types as B5V \& G2III and also concluded that the orbital period can undergo small unexpected changes and its behavior can be represented as a parabola indicating progressive decrease in orbital period. The component parameters were determined to be $\mathrm{M}_{h}=4.9 \mathrm{M}_{\odot}, \mathrm{M}_{c}=2.7 \mathrm{M}_{\odot}$, $\mathrm{R}_{h}=3.25 \mathrm{R}_{\odot}, \mathrm{R}_{c}=5.2 \mathrm{R}_{\odot}{ }^{3}$ and age is $\sim 3 \times 10^{5}$ years. Figure 27 represents $\mathrm{O}-\mathrm{C}$ variation obtained in the current study from quadratic ephemeris. The $(\mathrm{O}-\mathrm{C})$ plot shows the data points separated in two groups with no recorded observations of minima timings for about 38 years between the two groups. The first group has a scarce set of 5 data points and the next group is recognizably spread out with 23 data points. The polynomial fit to the available data shows a decrease in the period which is in accordance with that given by Zakirov (2001) and the obtained $\mathrm{dp} / \mathrm{dt}$ is $-3.0252 \times 10^{-6}$ days/year. Figure 28 represents the V light curve obtained from AAVSO. No additional work was carried out on this variable except for deriving new times of minima by Zejda (2004); Diethelm (2004); Kreiner (2004). It is further listed in eclipsing binary catalogues by Bud- 
ding et al. (2004); Malkov et al. (2006); Avvakumova et al. (2013).

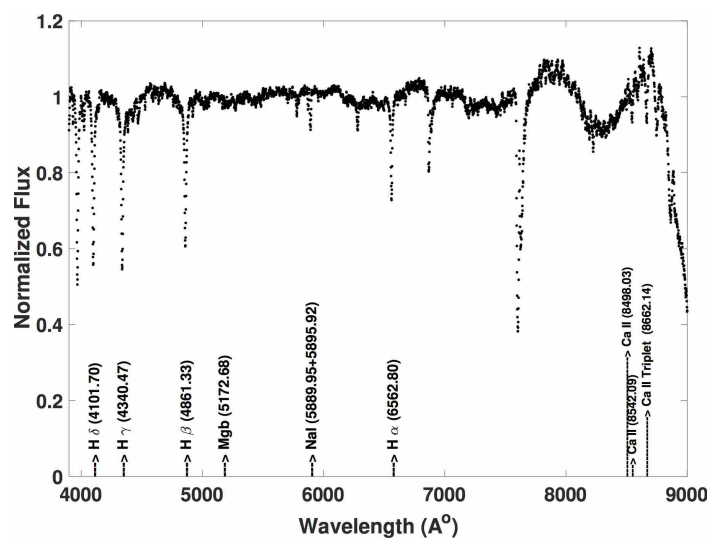

Fig. 29a Spectrum of BZ Mon (2013).

In the present study, the new ephemeris obtained is as follows HJD $($ Min I $)=2448660.263+3^{d} .451800 \times \mathrm{E}$.

In the present work two spectra were obtained for this variable on Feb 20, 2013 and Feb 20, 2014 at phases 0.070 (closer to primary minima), 0.807 (closer to maxima) respectively. These phases were calculated through the new epoch derived from period study. The spectral lines identified are shown in Figure 29 for the observed phases.There is an increase in the equivalent widths of Balmer lines near maxima compared to the spectrum obtained within the eclipse which shows spectral shallow due to filled-in absorption (as shown in Table 2). This can be attributed to the model defined by Zakirov (2001) where the period decrease was explained to be the result of non conservative mass flow and mass loss by the system. Avvakumova et al. (2013) have derived the spectral type to be B7 V. The best fit spectral model was selected (Jacoby et al. (1984)) to determine the spectral class as A7 $\mathrm{V}$, which has to be further validated.

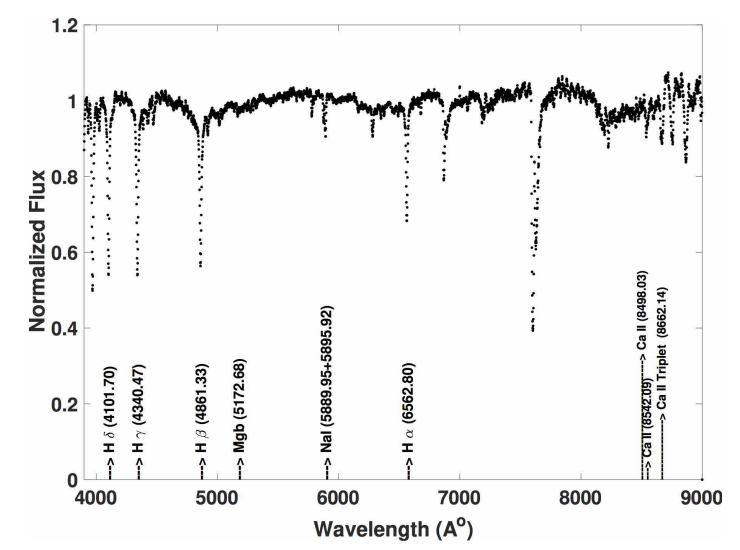

Fig. 29b Spectrum of BZ Mon (2014).

\footnotetext{
${ }^{3} \mathrm{~h}$ - hotter component, c - cooler component
}

\section{$3.17 \mathrm{CH}$ Mon}

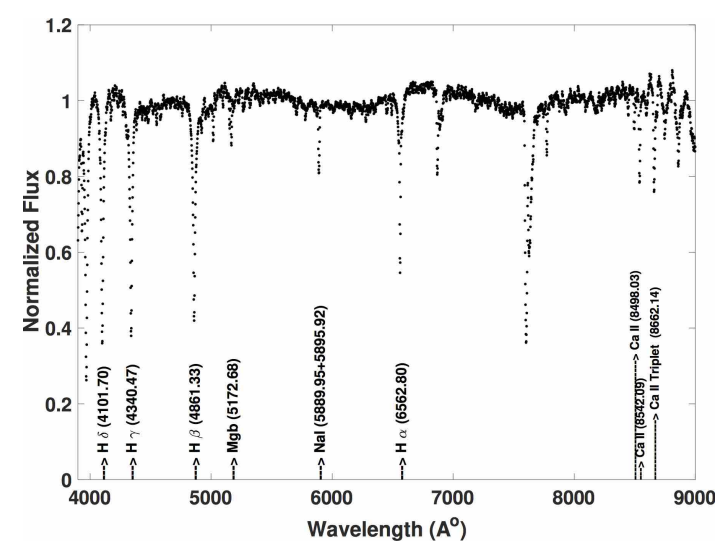

Fig. 30 Spectrum of CH Mon.

CH Mon(=AN 73.1936, GSC 00160-00530,B=13.1) was first reported as a variable by Hoffmeister (1936) and was further catalogued as an Algol type by Ahnert et al. (1947a); Kinnunen and Skiff (2000); Budding et al. (2004) and Malkov et al. (2006) . The period of the variable was determined to be $6^{d} .922312$ by Avvakumova et al. (2013) and no further study on times of minima or period variation was carried out in the literature. For the first time we obtained a spectra for $\mathrm{CH}$ Mon on Nov 12, 2013 at phase 0.014. It is again one of the least studied Algol type binaries. The dominant spectral lines are shown in Figure 30 and their equivalent widths calculated are as given in Table 2. The $\mathrm{H} \alpha$ absorption profile is observed to be relatively less prominent than the other Balmer lines which could be due to fill in effect.The spectral type was determined to be A8 V (Jacoby et al. (1984) based on minimum res $^{2}$ corresponding to evolved secondary component.

\subsection{FW Mon}

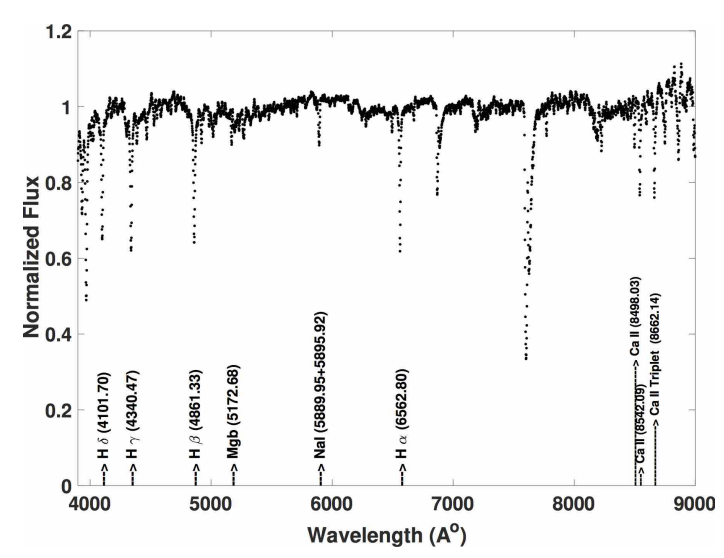

Fig. 31 Spectrum of FW Mon. 
FW Mon (= GSC 04845-02526=4845-2526-1, V = 9.97) is an Algol-type eclipsing binary with an orbital period $3^{d} .8735900$ (Kukarkin et al. (1971)). Chang (1948) was the first to study the variability of FW Mon and report the spectral types of brighter and fainter components as B5 and F2. Brancewicz and Dworak (1980) have presented physical parameters of FW Mon. Srivastava and Kandpal (1993) have done photoelectric observations and no period variation was found. Later on Budding et al. (2004) has presented minima of FW Mon in the catalogue of $\mathrm{Al}$ gol type binary stars. In the current study we performed period variation study for 15 data points spanning over 31 years and one point separated by 42 years. The data points show a large scatter but the best polynomial fit gives a decreasing period, however additional observations are required to understand the change clearly. The rate of decrease of period dp/dt obtained is $-1.6204 \times 10^{-7}$ days/year and the new epoch obtained is HJD (Min I) = $2448630.672+3^{d} .873589 \times$ E. One spectra was obtained on March 18, 2013 at phase 0.003 derived using the latest times of minima in the literature. Figure 31 displays the identified spectral lines and the equivalent widths obtained are given in Table 2 The spectral class of the variable is determined to be A9 V from (Jacoby et al. (1984)) our spectral observation.

\subsection{HP Mon}

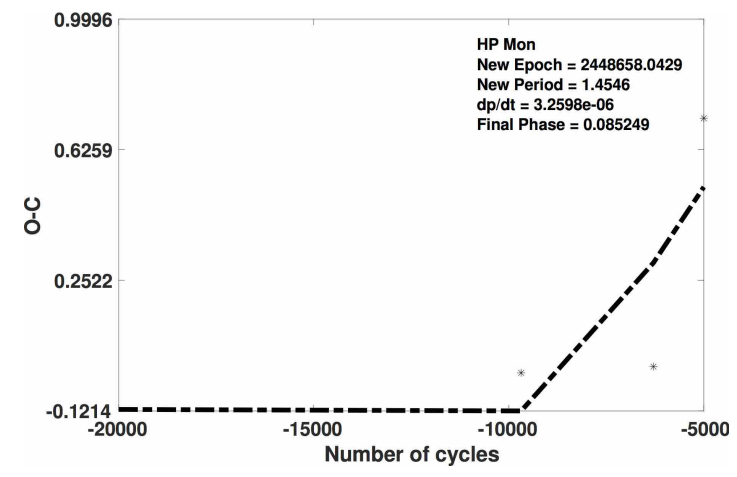

Fig. 32 O-C diagram of HP Mon.

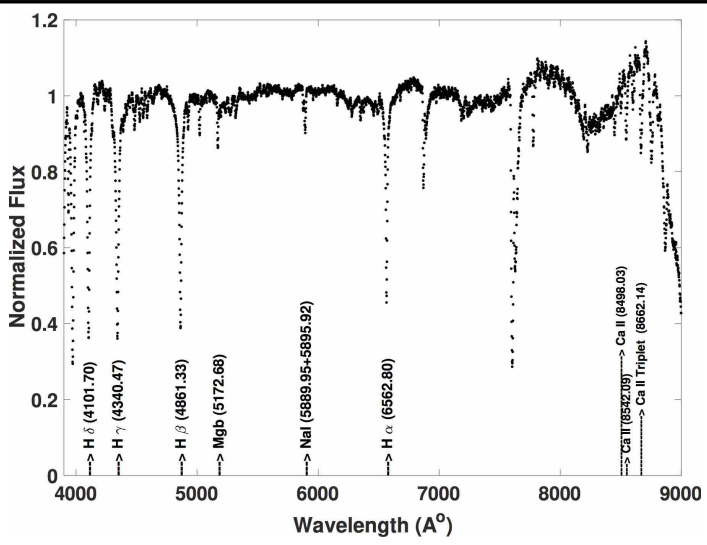

Fig. 33a Spectrum of HP Mon (2013).

HP Mon (= 2MASS J07103072-0533003) is an Algol-type eclipsing binary with an orbital period $1^{d} .45461$. It was listed in the catalogue of Algol type binary by Budding et al. (2004) and Malkov et al. (2006). Times of minima obtained through photoelectric observations were presented by Krajci 2006) and Hubscher et al. (2012). No period variation study was carried out so far, we present the first period study. This is another least studied Algol with only four data points available and spread across 70 years. The best fit curve shows increase in the period. The $\mathrm{O}-\mathrm{C}$ variation is shown in Figure 32 and $\mathrm{dp} / \mathrm{dt}$ obtained is $3.2598 \times 10^{-6}$ days/year. The new epoch obtained in the current study is as follows HJD (Min I) = $2456344.174+1^{d} .454641 \times \mathrm{E}$. Two spectra were obtained for this variable on Feb 20, 2013 and Feb 20, 2014 at phases $0.342,0.261$ respectively for the first time. The equivalent widths of the prominent spectral lines (Figure 33) are tabulated in Table 2. The best fit spectral model was selected from (Jacoby et al. (1984)) and spectral class is determined to be A5 V.

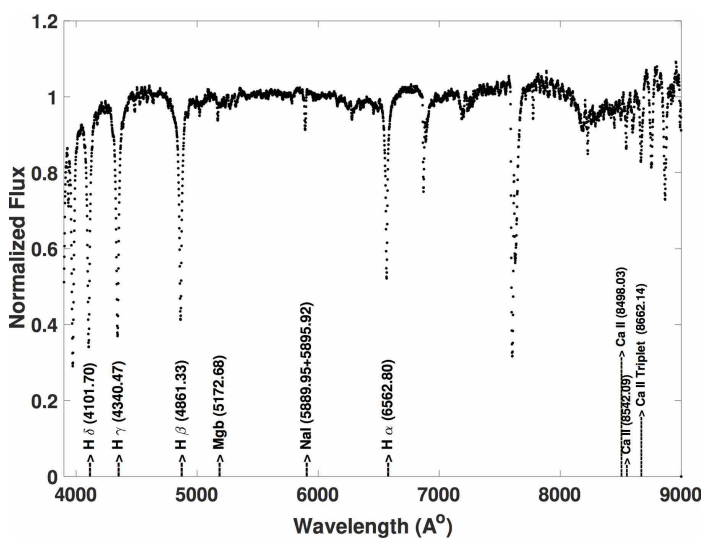

Fig. 33b Spectrum of HP Mon (2014). 


\section{$3.20 \mathrm{RV}$ Oph}

RV Oph (= 2MASS J16490246-1927524, V = 8.00) is an Algol-type eclipsing binary with an orbital period $3^{d}$ .687125. The variability of this star was first discovered by Mrs.Fleming (Pickering (1904)) and has been further observed by Dugan(1916). Walter (1970) carried out photoelectric observations of the variable in $\mathrm{B}$ and $\mathrm{V}$ bands. Compared to the previous observations the asymmetry was reduced and a model of gas stream was given explaining characteristics of light curves. Mezzetti et al. (1980) presented revised photometric light curves of RV Oph. From the photometric studies by Liao and Qian 2010 the light curve was found to be distinctly asymmetrical near primary minimum and irregularities in the phases out of eclipses were observed indicating influences of gas streams. The $(\mathrm{O}-\mathrm{C})$ has been plotted for 64 data points spanning over 45 years and one point separated by about 40 years. The data points show a large scatter but the best fit shows a decreasing period in Figure 34 with $\mathrm{dp} / \mathrm{dt}=-6.2191 \times 10^{-8}$ days/year. The new epoch deduced is HJD $($ Min I $)=2448634.730+3^{d} .6871 \times$ E. No spectroscopic observations were done till date. Thus, the first spectrum of the variable with a single spectrum observed on March 18, 2013 at phase 0.324 as derived from latest epoch in the literature. The spectral lines identified are given in Figure 35 and the equivalent widths are given in Table 2 It is observed that there is prominent fill in effect in $\mathrm{H} \alpha$ line when compared to other Balmer lines. Kreiner (1971) has given the spectral type of the variable as K0 whereas in the current study the best fit spectral model was selected from Jacoby et al. (1984) to determine the spectral class as F0 V which can be validated by further observations.

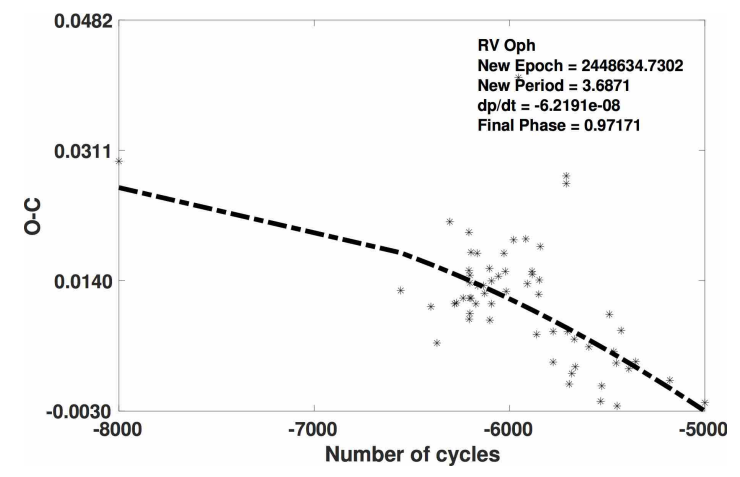

Fig. 34 O-C diagram of RV Oph.

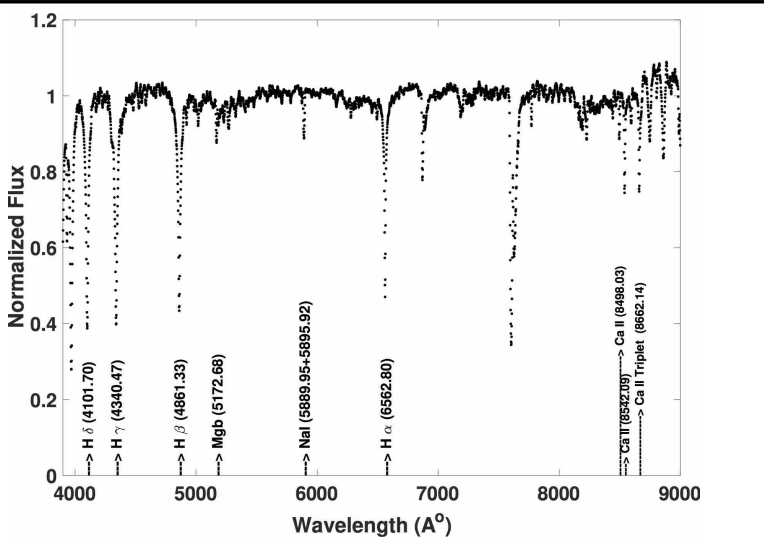

Fig. 35 Spectrum of RV Oph.

\subsection{FH Ori}

FH Ori(=GSC 00109-02559,TYC 109-2559-1,V=11.37) is a well studied Algol system with period of $2^{d} .151110$ Drake et al. (2014)). The variability of FH Ori was first detected by Hoffmeister (1929); Guthnick and Prager (1934) and the first photoelectric observations were done by Zakirov (1994) showing that FH Ori is a typical EA-type system. Many times of minima have been published (Kreiner (1971); Dworak (1977); Agerer and Huebscher (1998); Qian (2001)) to study period variations which indicated secular decrease in the orbital period. This was attributed to variable magnetic coupling and gravity coupling between the two components. They also determined that it is a variable with apsidal motion with high relative velocity and the orbital period is accounted to mass transfer from primary to secondary and spectroscopic studies were recommended for detailed results. We present first spectral study showing dominant profiles. The spectrum was obtained on Oct 14, 2013 at phase 0.2491 . The prominent spectral lines are given shown in Figure 36 and their equivalent widths in Table 2. The spectral type determined by Avvakumova et al. (2013) is A1V and by using the (Jacoby et al. (1984)) stellar library we determined it to be A2 V. 


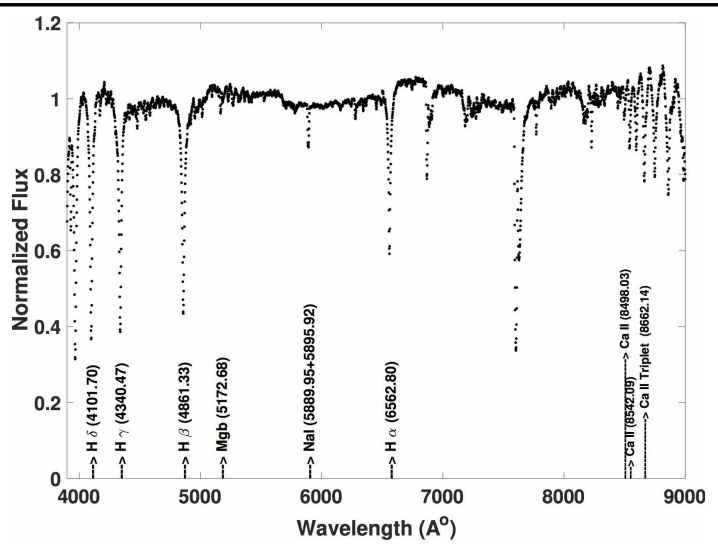

Fig. 36 Spectrum of FH Ori.

\subsection{Z Ori}

Z Ori (= GSC 00728-01172, TYC 728-1172-1, V=9.98) is one of the least studied Algol type binary. Its variability was first given by Gaposchkin (1932) and a spectrographic observations were carried out by Struve (1947) to determine the spectral type. The latest period available in the literature is $5^{d} .203265$ (Avvakumova et al. (2013)). Crawford (1955) categorized it to be a binary with subgiant secondary and spectral type as B5. Study for period variation was carried out by Prikhod'Ko (1962) who showed that the variation is cyclic. Very few minima are available in the literature to do period variation studies. One spectrum of Z Ori was obtained on Mar 21, 2014 at phase 0.9913 obtained from latest epoch available in the literature. The prominent spectral lines in the spectrum are shown in Figure 37 and their equivalent widths are given in Table 2. The spectrum displays absorption profiles of all the Balmer lines which could be due to evolved secondary component as seen from the phase. $\mathrm{H} \alpha$ line is weaker relative to other Balmer lines.

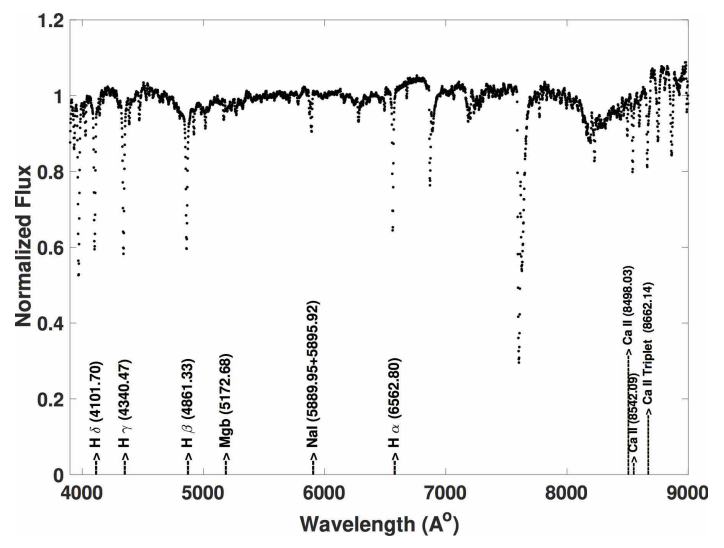

Fig. 37 Spectrum of Z Ori.

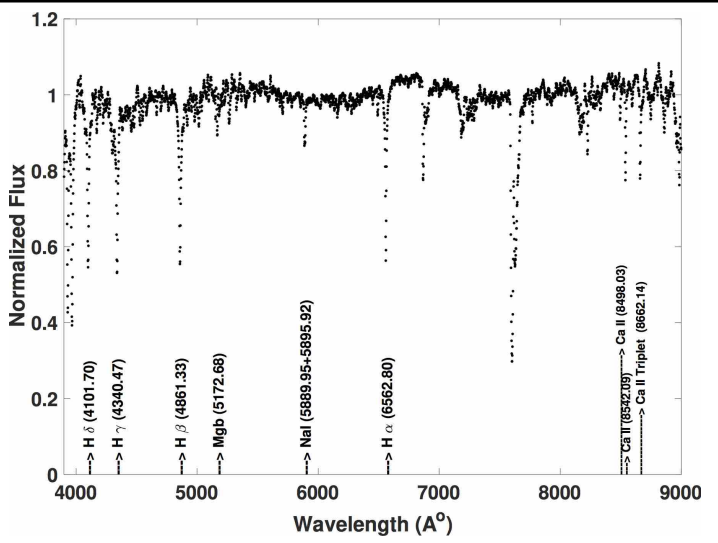

Fig. 38 Spectrum of V0640 Ori.

\subsection{V0640 Ori}

V0640 Ori (= GSC 05348-00080, TYC 5348-80-1, V=10.94) is one of the least studied Algol with a period of $2^{d} .020740$ (Avvakumova et al. (2013)). It was catalogued as an Algol type binary by Budding et al. (2004) and Malkov et al. (2006). Times of minima were given by many authors (Diethelm (2003, 2004); Dvorak 2004, 2005) and Locher (2005)). The times of minima in the literature are very few to carry out O-C studies. We obtained one spectrum for V0640 Ori on Oct 13, 2013 at phase 0.013 calculated with latest epoch in the literature. The dominant spectral lines are given in Figure 38 and equivalent widths are given in Table 2. The spectrum displays absorption profiles of all the Balmer lines which could be due to evolved secondary as seen from the phase. $\mathrm{H} \delta$ line shows weaker absorption profile relative to other Balmer lines. The best fit spectral model was found to be (Jacoby et al. (1984)) A3 III.

\subsection{CK Per}

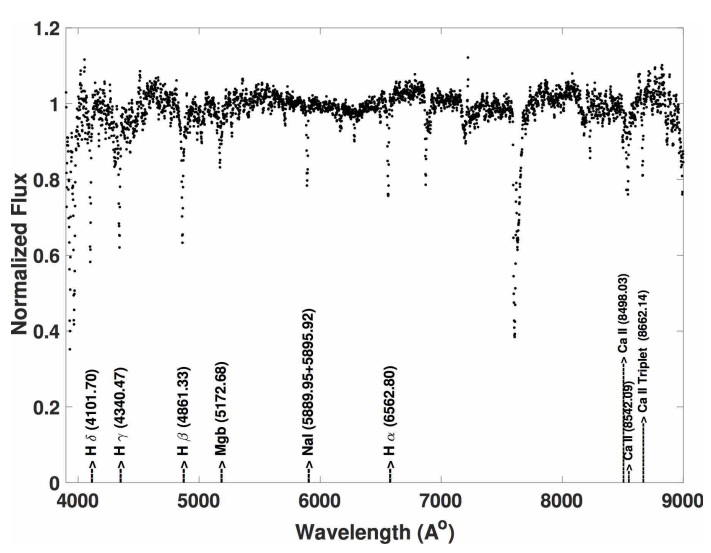

Fig. 39a Spectrum of CK Per (Oct,2013). 


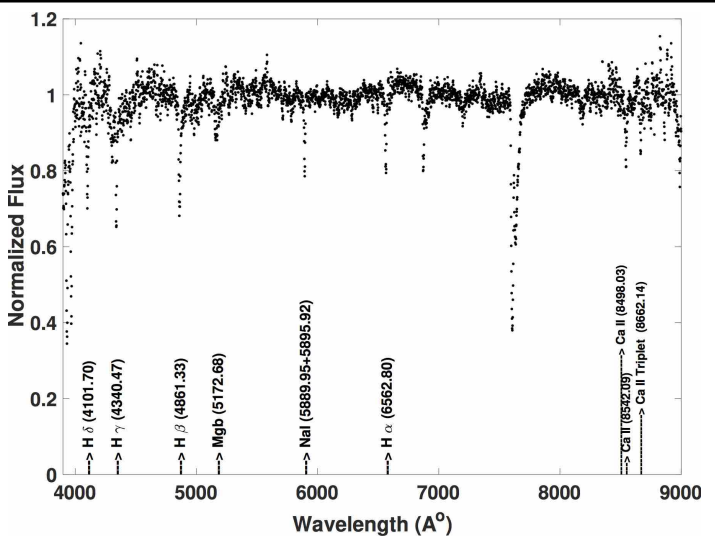

Fig. 39b Spectrum of CK Per (Nov,2013).

CK Per (AN 10.1940,2MASS J02050675+5610247, V=15.2) is one of the least studied Algol system. It was catalogued as a variable star by Ahnert et al. (1947a). The period of the variable is $2^{d} .372800$ (Kukarkin et al. (1971)) and was further catalogued as an Algol type by Budding et al. 2004); Malkov et al. 2006). Two spectra of CK Per were obtained on Oct 13 \& Nov 20, 2013 at phases 0.9948 \& 0.015 respectively for the first time. The dominant spectral lines are in Figure $39 \mathrm{~b}$ and equivalent widths are listed in Table 2. The $\mathrm{H} \delta$ line shows stronger absorption profile relative to other Balmer lines and also distinctly stronger absorption profile of $\mathrm{Na}$ line in one spectrum obtained in Oct 2013 where as it shows relatively weaker absorption profile in Nov 2013 near primary minima. This variable will be an interesting object for period study. Using (Jacoby et al. (1984)) stellar library, the spectral type is determined as F8 V.

\subsection{Z Per}

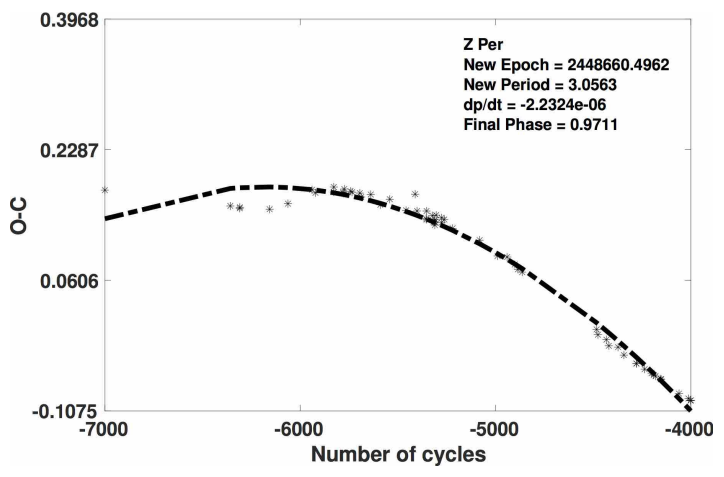

Fig. 40 O-C diagram of Z Per.

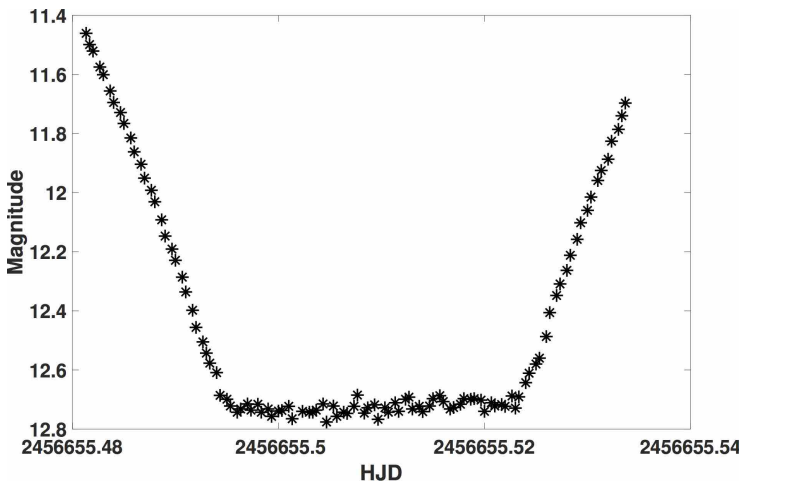

Fig. 41 Light curve of Z Per.

Z Per (= GSC 04147-01115 = TYC 4147-1115-1, V= 10.60) is an Algol-type eclipsing binary with an orbital period of $3^{d} .056306$. The orbital period of this Algol has been given by many authors (Wood and Forbes (1963); Mallama (1987)). These are about 61 ToM spread about 54 years and the best fit is distinctly representing a decreasing period. Many ToM were given in the literature and the variable was observed to have a decreasing period since the first AAVSO observations (Kreiner (1971); Samolyk (1997); Samolyk (2010)). Along with the secular decrease in orbital period, several sudden jumps were noticed in the orbital period of $\mathrm{Z}$ Per within a time interval of 94 years i.e, from early 1901 and late 1995. A sinusoidal variation is evident (Figure 40 and could be an unseen third star in the system with $\mathrm{dp} / \mathrm{dt}=-2.2324 \times 10^{-6}$ days/year. The O-C residuals obtained in the current study is as follows HJD $($ Min I $)=2456343.109+3^{d} .056283 \times$ E. The light curve obtained from the data available in the literature is shown in Figure 41 The spectral type of this variable is given as A0+G2 IV (Budding et al. (2004); Hoffman et al. (2006)).

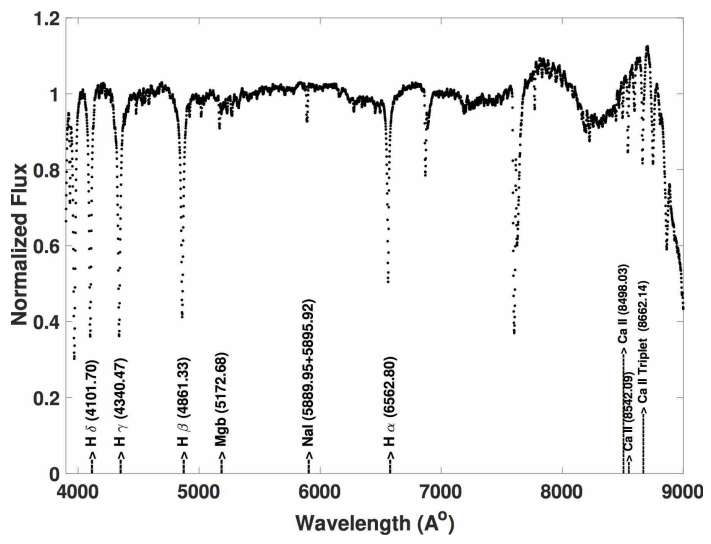

Fig. 42a Spectrum of Z Per (Feb, 2013). 


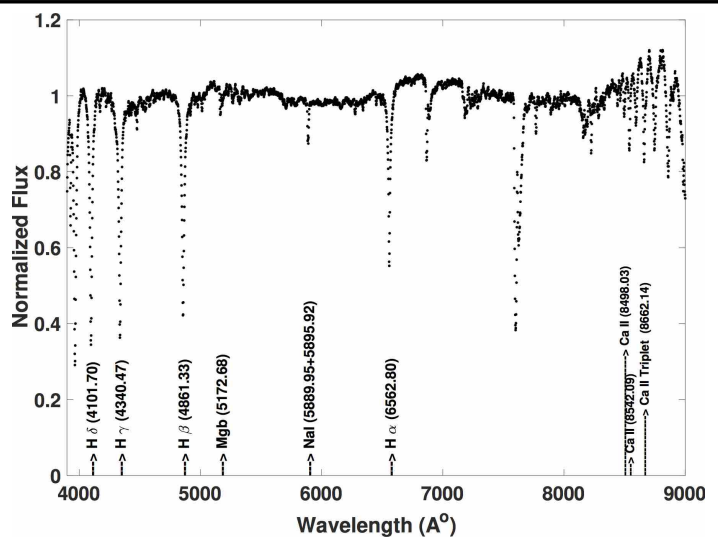

Fig. 42b Spectrum of Z Per (Oct, 2013).

We present the first spectral study of Z Per in this study. Three spectra were obtained for Z Per on Feb 20, 2013 at phase 0.015, Oct 13, 2013 at phase 0.016 and Feb 20, 2014 at phase 0.443 . The dominant spectral lines obtained are shown in Figure 42 and from the best fit spectral model (Jacoby et al. (1984)) the spectral class was determined as A7 V.

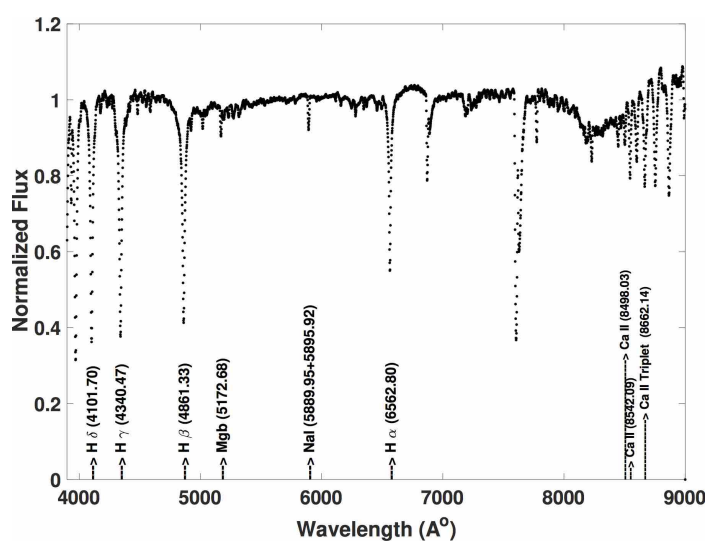

Fig. 42c Spectrum of Z Per (Feb, 2014).

\subsection{XY Pup}

XY Pup (=GSC 05421-00222, TYC 5421-222-1) was first discovered by Hoffmeister (1929). In the first spectral studies its spectral type was given as A3. Popper (1962); De Loore and Sutantyo (1984) also found two components at each of the D lines though not well separated. They also detected $\mathrm{H} \alpha$ absorption line to be blended with strong double emission attributed to the presence of circumbinary gaseous envelope photospheric matter. Brancewicz and Dworak (1980), classified the variable to be Algol type with spectral type A3e+K3 IV. The ToM were given in the literature by Dworak (1977); Dvorak (2004); Kreiner (2004). Extensive photometry was performed in the visible, IR \& Radio by Woodsworth and Hughes (1977); Kilkenny et al. (1985). Popper (1989) derived radial velocities from photometric analysis of $\mathrm{Na} D$ lines for cooler component and found evidence for circumstellar matter. The $\mathrm{H} \alpha$ line is in emission. It was also suggested that the velocities represented Pseudo elliptical motion. A spectrum was observed on Feb 20, 2013 and the phase obtained as 0.1956 using the epoch in the literature. The dominant spectral lines obtained are shown in Figure 43 and the equivalent width of the spectral lines are given in Table 2 in which sodium line shows greater equivalent width than all the other Algols under current study. As seen from the Table 2 the variable is distinctly characterized by emission spikes blended with absorption profile in Balmer lines which can be due to central emission (Vesper et al. 2001) and the double peaked emission is also suggestive of accretion disc.

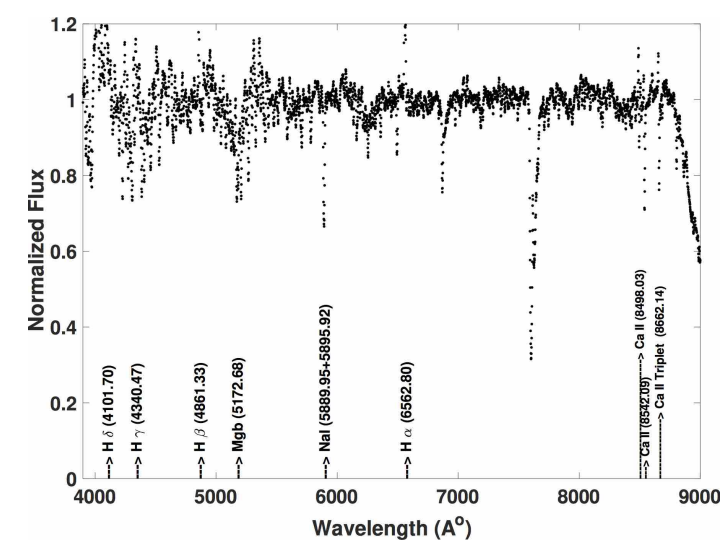

Fig. 43 Spectrum of XY Pup.

\subsection{AC Tau}

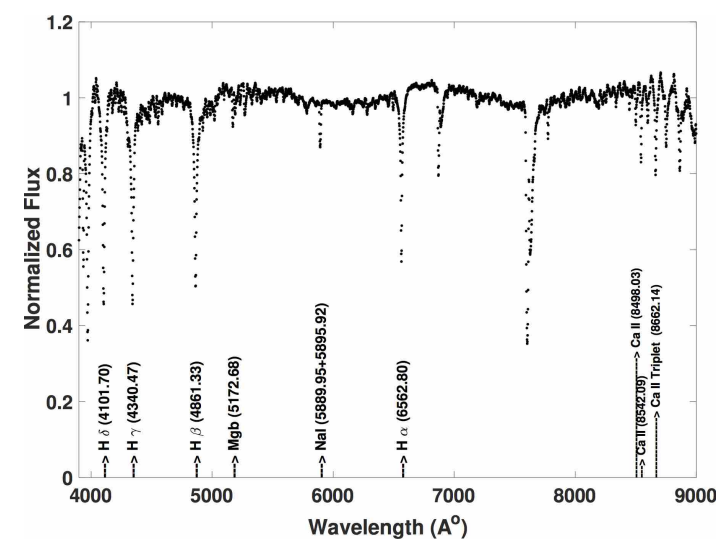

Fig. 44a Spectrum of AC Tau (Nov, 2013).

AC Tau(=AN 41.1929,GSC 00082-00147, V=11.09) is one of the least studied. It was catalogued as a variable star by 
Hoffmeister (1929); Webbink et al. (2002). The period of the variable is $2^{d} .043300$ as given by Samus et al. (2009). The spectral type was derived as F0+K6 by Baldwin and Samolyk (1997). The ROTSE data has given the O-C as 0.976 however the presence of third body for period variation was not asserted. Many ToM have been recorded by Wood and Forbes (1963); Kreiner (1976); Safar and Zejda (2000); Nelson (2003); Kreiner (2004); Locher (2005); Samolyk (2008). Qian (Qian (2000)) has compiled various times of light minimum and studied the changes in its orbital period which indicated cyclic variation with a secular increase. Pulsations have been defined and studied by Soydugan et al. 2006; Zhang et al. 2013) suggesting the delta scuti nature of the pulsations of the primary and a possible relation between pulsation and orbital period. In the current study we present one spectrum for AC Tau which was obtained on Dec 21, 2013 at phase 0.938 derived using latest epoch in the literature.

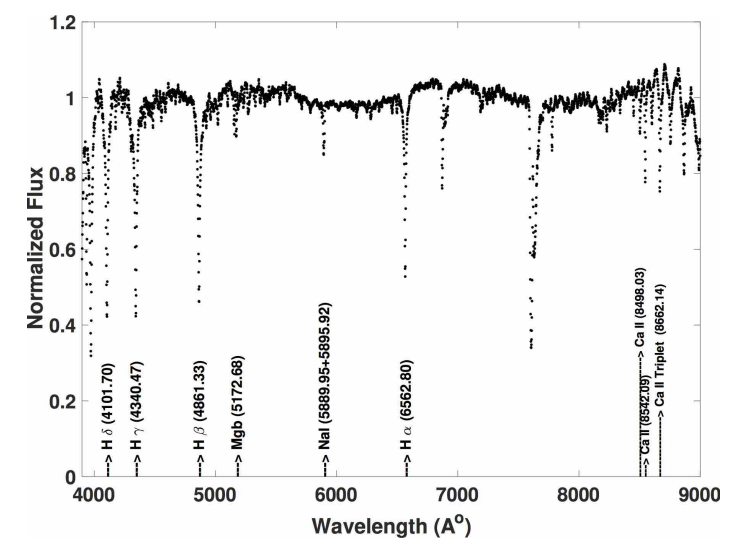

Fig. 44b Spectrum of AC Tau (Dec, 2013).

We present the dominant spectral lines in Figure 44 and their equivalent widths in Table 2. The spectrum displays absorption profiles of all the Balmer lines. The spectral class determined in this current study using best fit spectral model (Jacoby et al. (1984)) is F9 V.

\subsection{RW Tau}

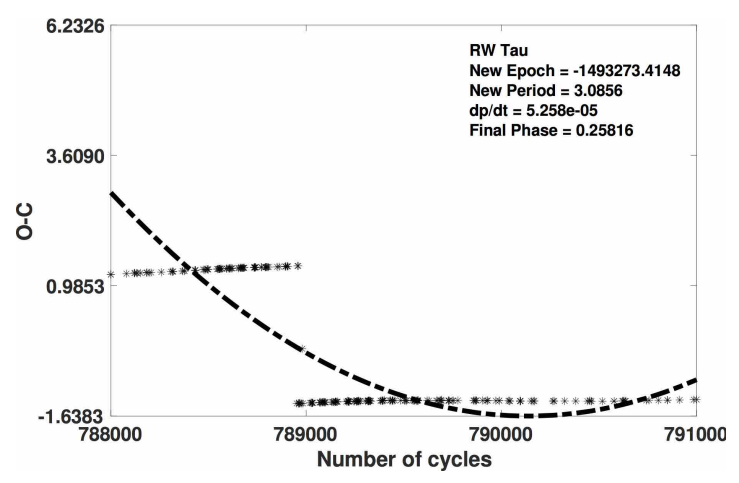

Fig. 45 O-C diagram of RW Tau.

RW Tau(=GSC 01826-00031=AN 102.1905, V=8.08) is a well studied Algol system with an orbital period of $2^{d} .76876$ belonging to spectral type B9V (Abt (2008)). It was first catalogued by Shapley (1913) and many ToM are given in the literature by various authors. The O-C variation obtained is shown in Figure 45 and the derived $\mathrm{dp} / \mathrm{dt}=5.25816 \times 10^{-8}$ days/year which represents a constant period. The new epoch obtained is as follows HJD $($ Min I $)=2456582.117+3^{d} .085628 \times$ E. Accretion disks were first observed in RW Tau by Wyse (1934) and Struve (1948, 1949) studied the rings in Algol system and suggested that the rings were due to accretion of gas from the secondary star.

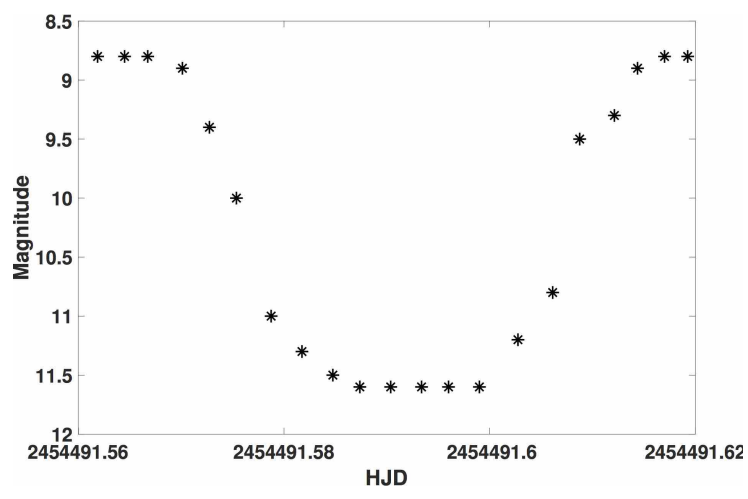

Fig. 46 Light curve of RW Tau. 
21

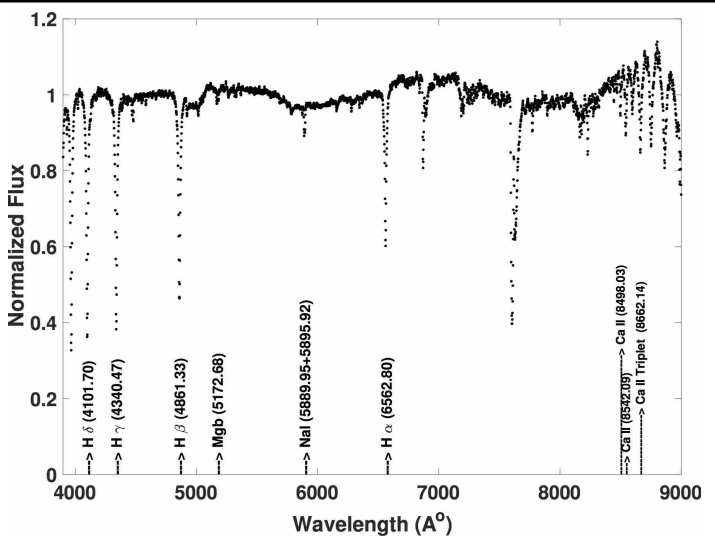

Fig. 47 Spectrum of RW Tau.

Kaitchuck and Honeycutt (1982) have done detailed study and computed disk radii from measured times of disappearance and reappearance of each doppler component during eclipse. RW Tau is well known for its transient disks and Lubow and Shu (1975) have calculated the gas stream trajectories. Kaitchuck et al. (1985) showed emission line widths to be almost constant during eclipse and twice as broad as expected from rotational broadening in a disk. One spectra on Oct 16, 2013 was observed for RW Tau at phase 0.115 . The prominent spectral lines are shown in Figure 47 and their equivalent widths are given in Table 2. The spectral type as obtained from the best fit spectral model using Jacoby et al. (1984) is B9 III which is similar to that obtained byJoy and Wilson (1949). As per the available models Lubow and Shu (1975) and Kaitchuck et al. (1985), RW Tau is observed to be showing primary radius larger than $\omega_{\text {disk }}$. This implies that a stable accretion disk could not form but possibility of circumstellar bulges exists. The light curve obtained from the data available in the literature is shown in Figure 46 The system may have circumbinary gaseous envelop.

\subsection{AF UMa}

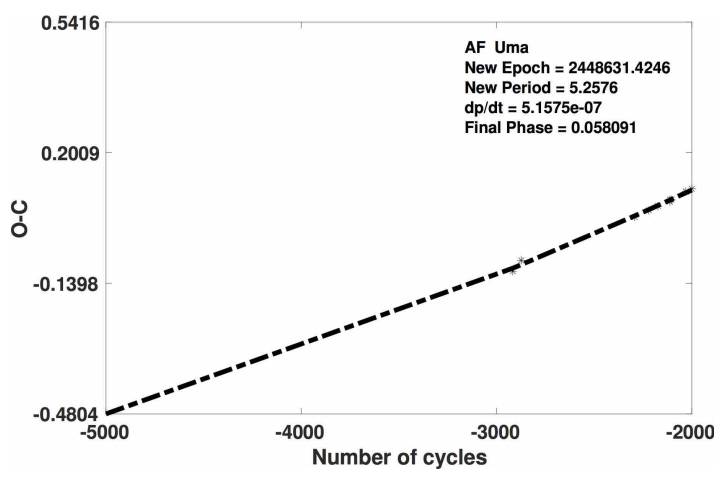

Fig. 48 O-C diagram of AF UMa.

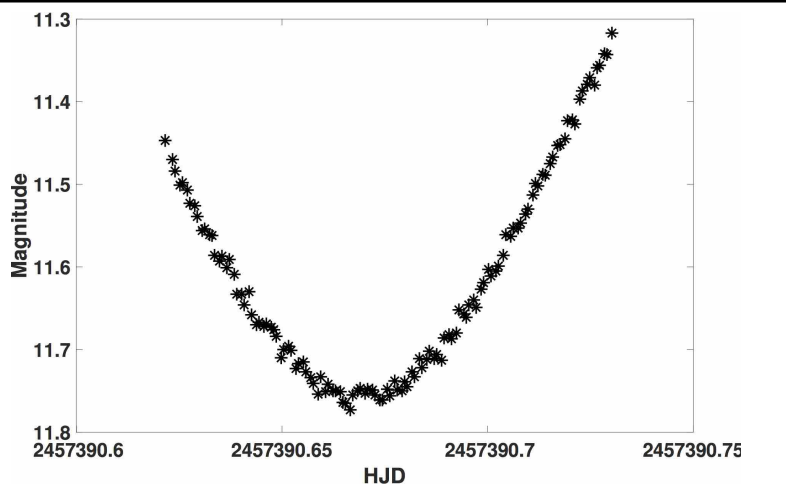

Fig. 49 Light curve of AF UMa.

AF UMa (= GSC 04147-01115 = TYC 4147-1115-1, V = 10.60 ) is an Algol-type eclipsing binary with an orbital period $5^{d} .25755$. No detailed study on the orbital period changes of the system has been done so far. Brancewicz and Dworak (1980) presented geometric and physical parameters of AF UMa. Many times of minima were reported in the literature by Malkov et al. (2006); Samolyk (2008, 2010, 2011); Brát et al. (2011). The photoelectric observations were carried out and the minima were given in IBVS by Hubscher et al. (2006); Hubscher et al. (2012, 2015, 2016). The spectral type of the variable is A0 (Brancewicz and Dworak (1980)). The O-C diagram is shown in Figure 48 which has been plotted for only 11 data points spanning over 28 years and one point separated by 57 years. The parabolic relation shows a tight fit for the observed data, indicating an increase in the period. However, this information can be authenticated with further observations. Using the quadratic term found in the $\mathrm{O}-\mathrm{C}$ analysis, the increase rate in the period of AF UMa is derived to be about $\mathrm{dp} / \mathrm{dt}=5.1575 \times 10^{-7}$ days/year.

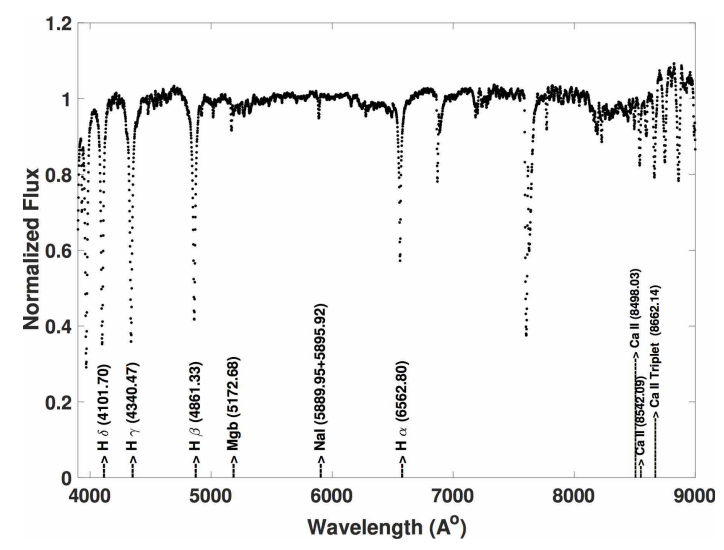

Fig. 50a Spectrum of AF UMa (Mar, 2013). 


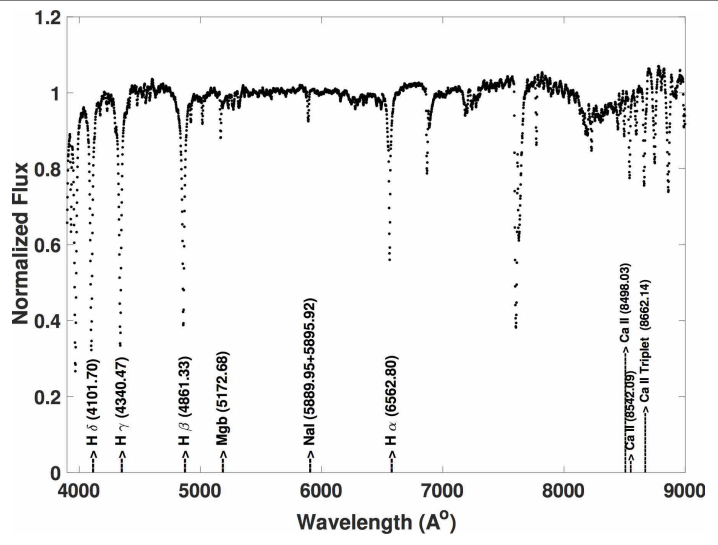

Fig. 50b Spectrum of AF UMa (Mar, 2014).

The new ephemeris is obtained to be as HJD $($ Min I $)=$ $2448631.4246+5^{d} .2576 \times$ E. Figure 49 shows the V-light curve from the observational data taken from AAVSO. Two spectra of AF UMa were observed on Mar 18, 2013 and Mar 21, 2014. The spectra obtained were at phases 0.942 and 0.940 respectively which were calculated using the new epoch derived from period study. The spectral lines identified are shown in Figure 50 It is observed that there is an increase in the equivalent widths of the Balmer lines (as shown in Table 2) especially in the $\mathrm{H} \alpha$ absorption line. AF UMa falls in to category 1A (Figure 52) of model given by Vesper et al. (2001) which signifies a characteristic of symmetric disk or bulge but no direct indications of stream disk interaction. As per the models available Lubow and Shu (1975); Kaitchuck (1985), the position of AF UMa is observed to be showing primary radius greater than $\omega_{\text {disk }}$ but smaller than $\omega_{\text {min }}$. This implies that the accretion stream would have interacted with the primary component and formed into a stable disk as evident from the absorption line profiles observed in the spectra. The best estimate of spectral type for AF UMa based on minimum res ${ }^{2}$ and visual inspections is derived to be A7 V using Jacoby et al. (1984) stellar library. The spectral type derived is varying from that reported earlier which could be due to phase dependance.

\section{$3.30 \mathrm{VV}$ Vul}

VV Vul (=TYC 2180-593-1, V=12.40) was first discovered by Wolf (1904) while searching for variables in Halbedel (1984) derived the spectral class to be $\mathrm{A} 2 / 3 \mathrm{~V}$ and the period was derived as $3^{d} .411400$ by Kreiner (2004). Few times of minima were recorded between $1904 \& 2009$ and were catalogued by Budding et al. (2004); Malkov et al. (2006) and the period changes were determined by Qian (2000); Qian et al. (2002). O-C studies done by Qian showed a secular increase in the period and from the assumed parameters gave a high rate of mass transfer. In the current study a spectrum for VV Vul was obtained at phase 0.868 on Nov 20, 2013. The dominant profiles in the spectra are shown in Figure 51 and equivalent widths derived are given in Table 2

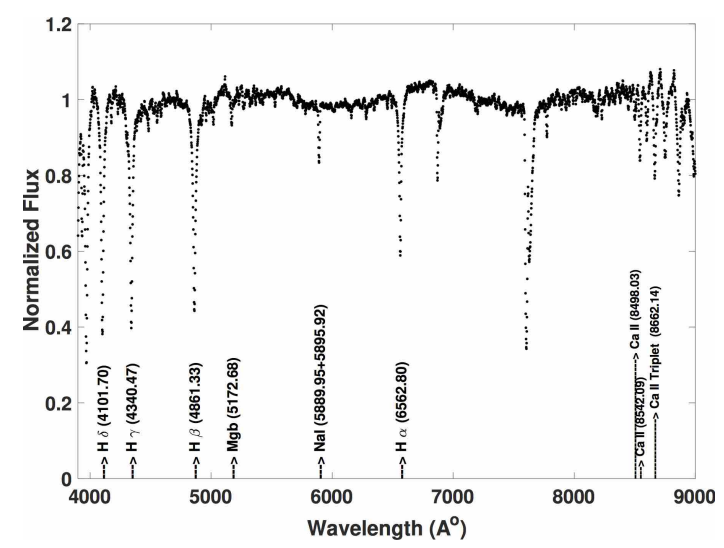

Fig. 51 Spectrum of VV Vul.

\section{Conclusions}

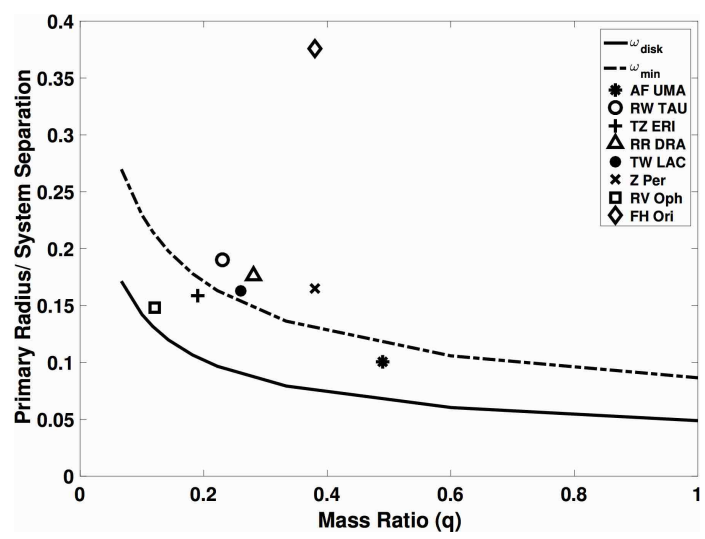

Fig. 52 A plot of primary radius vs mass ratio as per the model given by Lubow and Shu (1975); Kaitchuck (1985); Vesper et al. (2001).

The dominant spectral lines in all the Algols were observed to be of $\mathrm{H} \delta, \mathrm{H} \gamma, \mathrm{H} \beta, \mathrm{H} \alpha, \mathrm{Na}$ I, Ca II \& Ca triplet with absorption profile except for XY Pup in which emission spike in $\mathrm{H}$ lines and absorption in Na line is observed to be dominant. These systems are listed in Table 1 in alphabetical order along with details of observations and we report the equivalent widths (EWs) obtained and observed features in Table2. For the variables AF UMa, RW Tau, TZ Eri, RR Dra, TW Lac, Z Per, RV Oph \& FH Ori we tried to examine the systems with the model of Lubow and Shu (1975) who established two parameters $\omega_{\min }$ and $\omega_{\text {disk }}$ that indicate the characteristics of accretion 
disk. They signify the stream trajectory and disk formation in the orbit plane as a function of mass ratio. It was observed that none of the systems had parameters below the $\omega_{\min }$ line which signifies that the systems stream misses the primary component and could form a stable accretion disk. The spectral profiles obtained indicated clear absorption lines.

We checked the physical parameters ( $\mathrm{T}_{e f f}$ and log g) for the Algols in this study and this task was accomplished by fitting model atmosphere spectra Jacoby et al. (1984) to the observed spectra and the spectral types were derived. Using the equivalent widths obtained for $\mathrm{H} \gamma$ line we tried to derive $\log g$ values for the Algols as seen in Figure 53 as $\mathrm{H} \gamma$ line is considered sensitive to gravity and temperature thus making it an effective tool in determining the fundamental stellar parameters (Martins et al. (2005)).

The G-band extended around $4300 \AA$ is used as an approximate measure of $\mathrm{CH}$ abundance, make an appearance around F3 and become very strong at late G-type to K-type (Morgan et al. (1943)). The variables V 769 Aql, XY Cet, RR Dra, SX Gem, FG Lyr, CH Mon, FW Mon, Z Ori, V 640 Ori, CK Per, Z Per for which the spectra were obtained at phases close to zero, indicate relatively weak G-band. High resolution spectra during totality phase are needed to confirm and derive carbon abundance (Parthasarathy et al. (1983)). This was further validated by comparing the G-band absorption profiles with that of $\mathrm{H} \gamma$ absorption lines. For many Algols in our sample there are no recent observed epochs of primary minima, therefore the phases given in Table 1 may not be accurate.

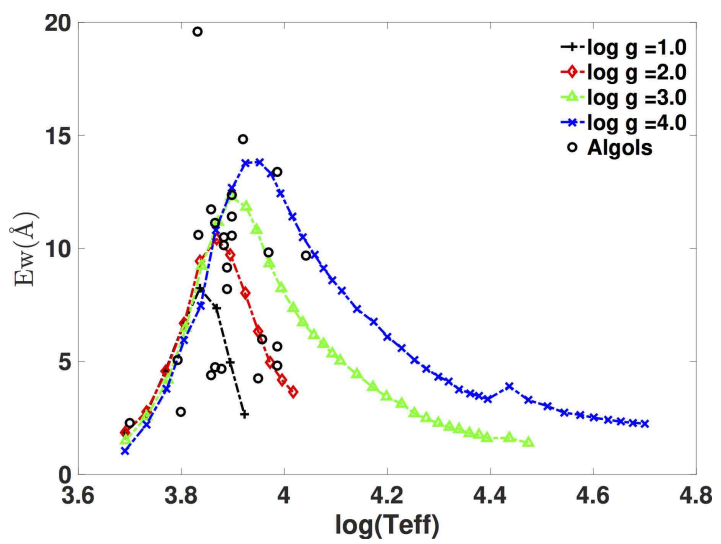

Fig. 53 Equivalent width of $\mathrm{H} \gamma$ as a function of the effective temperature adopted from Martin (Martins et al. (2005) and Algols in study overplotted to derive log g.

The H-alpha line in the spectrum of several systems in our sample is relatively weak and or partly filled in which indicates mass transfer and or circumstellar gas. Monitoring the $\mathrm{H}$-alpha profile of these systems at high resolution can reveal more information. Wavelength region more than $8000 \AA$ and in the Ca II triplet lines the blending of the primary star spectrum with the flux of the late-type secondary can be significant. The late-type secondary components in Algol-type systems seems to have strong chromospheric activity than single stars of similar spectral type therefore the Ca II IR triplet strengths can be weaker (Parthasarathy et al. (1979)). High resolution and high signal to noise ratio spectra at 0.25 and 0.75 phases of most of the system in our sample may reveal the $\mathrm{H}$ alpha line of the secondary component (see for example Parthasarathy (2017) and Parthasarathy (2018)). Algoltype semidetached systems in which the primary minimum showing long duration flat bottom totality phase similar to that of Z Per (see Figure 42 are well suited for obtaining uncontaminated high resolution and high signal to noise ratio spectra of the late-type evolved low mass secondary component to derive its detailed chemical composition including, C, N, O and S-process elements and may be even $\mathrm{C} 12 / \mathrm{C} 13$ isotope ratio. Such a study will enable us to understand the nucleosynthesis, mixing, mass-transfer, mass-loss and evolution process experienced by Algol-type close binaries.

\section{Acknowledgement}

We are thankful to Prof. G. C. Anupama for helping us to get the observational data with the Himalayan Chandra Telescope (HCT). We are very grateful to Dr. Oleg Malkov for his encouraging comments. 


\section{References}

Abt, H.A.: The Astrophysical Journal Supplement Series 180(1), 117 (2008)

Agerer, F., Hubscher, J.: Information Bulletin on Variable Stars 4383 (1996)

Agerer, F., Hubscher, J.: Information Bulletin on Variable Stars 5484 (2003)

Agerer, F., Hubscher, J.: Information Bulletin on Variable Stars 5016 (2001)

Agerer, F., Huebscher, J.: Information Bulletin on Variable Stars (1998)

Ahnert, P., Hoffmeister, C.: Kl. Ver. Bebelsberg 28, 1 (1943)

Ahnert, P., Hoffmeister, C., Rohlfs, E., van de Voorde, A.: Veroeffentlichungen der Sternwarte Sonneberg 1, 43 (1947a)

Ahnert, P., Hoffmeister, C., Rohlfs, E., van de Voorde, A.: Veroeffentlichungen der Sternwarte Sonneberg 1, 43 (1947b)

Arnold, C.N., Hall, D.S.: Information Bulletin on Variable Stars 843 (1973)

Avvakumova, E., Malkov, O.Y., Kniazev, A.Y.: Astronomische Nachrichten 334(8), 860 (2013)

Baldwin, M., Samolyk, G.: MA: AAVSO (4) (1997)

Barblan, F., Bartholdi, P., North, P., Burki, G., Olson, E.: Astronomy and Astrophysics Supplement Series 132(3), 367 (1998)

Borovicka, J.: Astronomy and Astrophysics 279, 627 (1993)

Brancewicz, H., Dworak, T.Z.: Acta Astronomica 30, 501 (1980)

Brát, L., Trnka, J., Smelcer, L., Lehky, M., Kucakova, H., Lomoz, F, Hanzl, D., Vrastak, M., Corfini, G., Pribik, V., et al.: Open European Journal on Variable Stars 137, 1 (2011)

Brát, L., Zejda, M., Svoboda, P.: OEJV 74, 1 (2007)

Budding, E., Erdem, A., Çiçek, C., Bulut, I., Soydugan, F., Soydugan, E., Bakış, V., Demircan, O.: Astronomy \& Astrophysics 417(1), 263 (2004)

Cannon, A.J.: Harvard College Observatory Bulletin 897, 12 (1934)

Catalano, S., Rodono, M.: Publications of the Astronomical Society of the Pacific 86(512), 390 (1974)

Chang, Y.: The Astrophysical Journal 107, 96 (1948)

Christopoulou, P.-E., Papageorgiou, A., Kleidis, S., Tsantilas, S.: The Astronomical Journal 143(2), 30 (2011)

Crawford, J.: The Astrophysical Journal 121, 71 (1955)

Cutri, R., Skrutskie, M., Van Dyk, S., Beichman, C., Carpenter, J., Chester, T., Cambresy, L., Evans, T., Fowler, J., Gizis, J., et al.: VizieR Online Data Catalog 2246 (2003)

De Loore, C.: In: International Astronomical Union Colloquium, vol. 80, p. 199 (1984). Cambridge University Press

De Loore, C., Sutantyo, W.: Astrophysics and Space Science 99, 335 (1984)

Diethelm, R.: Information Bulletin on Variable Stars 5438 (2003)

Diethelm, R.: Information Bulletin on Variable Stars 5543 (2004)

Diethelm, R.: Information Bulletin on Variable Stars 5653, 33 (2005)

Dogru, S.S., Donmez, A., Tuysuz, M., Dogru, D., Ozkardes, B., Soydugan, E., Soydugan, F.: Information Bulletin on Variable Stars 5746, 1 (2007)
Drake, A., Graham, M., Djorgovski, S., Catelan, M., Mahabal, A., Torrealba, G., García-Álvarez, D., Donalek, C., Prieto, J., Williams, R., et al.: The Astrophysical Journal Supplement Series 213(1), 9 (2014)

Dugan, R.S., Wright, F.W.: Contributions from the Princeton University Observatory 19, 34 (1939)

Dugan, R.: The Astrophysical Journal 43 (1916)

Dvorak, S.: Communications in Asteroseismology 160, 64 (2009)

Dvorak, S.W.: Information Bulletin on Variable Stars $\mathbf{5 5 0 2}$ (2004)

Dvorak, S.W.: Information Bulletin on Variable Stars 5603 (2005)

Dworak, T.: Acta Astronomica 27, 151 (1977)

Eker, Z., Bilir, S., Soydugan, F., Gökçe, E.Y., Soydugan, E., Tüysüz, M., Şenyüz, T., Demircan, O.: Publications of the Astronomical Society of Australia 31 (2014)

Eker, Z., Soydugan, F., Soydugan, E., Bilir, S., Gökçe, E.Y., Steer, I., Tüysüz, M., Şenyüz, T., Demircan, O.: The Astronomical Journal 149(4), 131 (2015)

Erdem, A., Soydugan, F., Doğru, S., Özkardeş, B., Doğru, D., Tüysüz, M., Demircan, O.: New Astronomy 12(8), 613 (2007)

Faulkner, D., Kaitchuck, R.: Information Bulletin on Variable Stars 2321 (1983)

Fernández-Figueroa, M., Montes, D., De Castro, E.: The Astrophysical Journal Supplement Series 90, 433 (1994)

Fernández-Figueroa, M., Barrado, D., De Castro, E., Cornide, M.: Astronomy and Astrophysics 274, 373 (1993)

Frasca, A., Lanza, A.: Astronomy \& Astrophysics 429(1), 309 (2005)

Gaposchkin, S.: Veröffentlichungen der Universitätsstern-warte zu Berlin-Babelsberg 5 (1932)

Giuricin, G., Mardirossian, F., Mezzetti, M.: The Astrophysical Journal Supplement Series 52, 35 (1983)

Giuricin, G., Mardirossian, F., Mezzetti, M.: The Astrophysical Journal Supplement Series 54, 421 (1984)

Guthnick, P., Prager, R.: Astronomische Nachrichten 251(17), 257 (1934)

Halbedel, E.: Information Bulletin on Variable Stars 2549 (1984)

Hall, D.S.: Publications of the Astronomical Society of the Pacific 84(498), 323 (1972)

Harmanec, P.: Bulletin of the Astronomical Institutes of Czechoslovakia 39, 329 (1988)

Hoffman, D., Harrison, T., McNamara, B., Vestrand, W., Holtzman, J., Barker, T.: The Astronomical Journal 132(6), 2260 (2006)

Hoffmeister, C.: Astronomische Nachrichten 233, 33 (1928)

Hoffmeister, C.: Astronomische Nachrichten 236, 233 (1929)

Hoffmeister, C.: Astronomische Nachrichten 200(11), 177 (1914)

Hoffmeister, C.: Astronomische Nachrichten 201(23), 443 (1915)

Hoffmeister, C.: Astronomische Nachrichten 240(12), 193 (1930)

Hoffmeister, C.: Astronomische Nachrichten 258(2), 25 (1936)

Hoffmeister, C.: Astronomische Nachrichten 274(4), 176 (1943)

Hubscher, J.: Information Bulletin on Variable Stars $\mathbf{5 8 0 2}$ (2007) 
Hubscher, J., Paschke, A., Walter, F.: Information Bulletin on Variable Stars 5731 (2006)

Hubscher, J.: Information Bulletin on Variable Stars 5643, 1 (2005)

Hubscher, J., Lehmann, P.B., Walter, F.: Information Bulletin on Variable Stars 6010 (2012)

Hubscher, J., Lehmann, P.B., Walter, E.: Information Bulletin on Variable Stars 6152 (2015)

Hubscher, J., Lehmann, P.B., Walter, F.: Information Bulletin on Variable Stars 6157 (2016)

Hubscher, J., Steinbach, H.-M., Walter, F.: Information Bulletin on Variable Stars 5874 (2009)

Hübscher, J., Lehmann, P.B., Monninger, G., Steinbach, H., Walter, F: IBVS 5889, 1 (2013)

Jacoby, G.H., Hunter, D.A., Christian, C.A.: The Astrophysical Journal Supplement Series 56, 257 (1984)

Joy, A.: Publications of the Astronomical Society of the Pacific 34, 221 (1922)

Joy, A.H., Wilson, R.E.: The Astrophysical Journal 109, 231 (1949)

Kaitchuck, R.H.: Publication of the Astronomical Society of the Pacific 97, 1178 (1985)

Kaitchuck, R.H., Honeycutt, R.K.: Publications of the Astronomical Society of the Pacific 94(559), 532 (1982)

Kaitchuck, R.H., Park, E.A.: The Astrophysical Journal 325, 225 (1988)

Kaitchuck, R.H., Honeycutt, R.K., Schlegel, E.M.: Publications of the Astronomical Society of the Pacific 97(598), 1178 (1985)

Keller, G., Limber, D.: The Astrophysical Journal 113, 637 (1951)

Kholopov, P., Samus', N., Kukarkina, N., Medvedeva, G., Perova, N.: Information Bulletin on Variable Stars 1921 (1981)

Kilkenny, D., Whittet, D.C.B., Davies, J.K., Evans, A., Bode, M.F., Robson, E.I., Banfield, R.M.: South African Astronomical Observatory Circular 9 (1985)

Kinnunen, T., Skiff, B.A.: Information Bulletin on Variable Stars 4863 (2000)

Kopal, Z.: In: Annales d'Astrophysique, vol. 18, p. 379 (1955)

Kordylewski, K.: Ron. Astron. SAC 31, 133 (1959)

Kordylewski, K.: Information Bulletin on Variable Stars 35 (1963)

Krajci, T.: Information Bulletin on Variable Stars 5690, 1 (2006)

Kreiner, J.M.: Acta Astronomica 21, 365 (1971)

Kreiner, J.M.: Acta Astronomica 54, 207 (2004)

Kreiner, J.: Acta Astronomica 26, 341 (1976)

Kukarkin, B.V., Kholopov, P.N., Efremov, Y.N., Kukarkina, N.P., Kurochkin, N.E., Medvedeva, G.I., Perova, N.B., Pskovskij, Y.P., Fedorovich, V.P., Frolov, M.S.: Astronomical Council of the Academy of Sciences of the USSR, 324 (1971)

Kurochkin, N.E.: Peremennye Zvezdy 10, 171 (1954)

Kurochkin, N.: Peremennye Zvezdy Prilozhenie 3, 415 (1979)

Lanza, A., Rodonò, M.: Astronomische Nachrichten 325(5), 393 (2004)

Liakos, A., Niarchos, P.: Communications in Asteroseismology 160, 2 (2009)

Liao, W.-P., Qian, S.-B.: Monthly Notices of the Royal Astronomical Society 405(3), 1930 (2010)

Locher, K.: Open European Journal on Variable Stars 3, 1 (2005)
Lubow, S.H., Shu, F.H.: The Astrophysical Journal 198, 383 (1975)

Malkov, O.Y., Oblak, E., Snegireva, E., Torra, J.: Astronomy \& Astrophysics 446(2), 785 (2006)

Mallama, A.: Journal of the American Association of Variable Star Observers (JAAVSO) 16, 4 (1987)

Mallama, A.: The Astrophysical Journal Supplement Series 44, 241 (1980)

Martins, L., Delgado, R.M.G., Leitherer, C., Cerviño, M., Hauschildt, P.: Monthly Notices of the Royal Astronomical Society 358(1), 49 (2005)

Meinunger, L.: W. Strohmeier, R. Knigge, H. Ott (1965)

Mezzetti, M., Cester, B., Giuricin, G., Mardirossian, F.: Astronomy and Astrophysics Supplement Series 39, 273 (1980)

Mkrtichian, D., Rodríguez, E., Olson, E., Kusakin, A., Kim, S.-L., Lehmann, H., Gamarova, A.Y., Kang, Y.: In: Tidal Evolution and Oscillations in Binary Stars, vol. 333, p. 197 (2005)

Morgan, W.W., Keeman, P.C., Kellman, E.: An Atlas of Stellar Spectra,. University of Chicago press Chicago, ??? (1943)

Morrison, N.D., Morrison, D.: The Astronomical Journal 73, 28 (1968)

Nelson, R.H.: Information Bulletin on Variable Stars 5371 (2003)

Okazaki, A.: Astrophysics and Space Science 56(2), 293 (1978)

Parthasarathy, M.: ArXiv e-prints: arXiv170706332 (2017)

Parthasarathy, M.: Information Bulletin on Variable Stars 63(6233 (In-press)) (2018)

Parthasarathy, M., Lambert, D.L., Tomkin, J.: Monthly Notices of the Royal Astronomical Society 186(3), 391 (1979)

Parthasarathy, M., Lambert, D., Tomkin, J.: Monthly Notices of the Royal Astronomical Society 203(4), 1063 (1983)

Pickering, E.C.: Astronomische Nachrichten 166, 39 (1904)

Plavec, M., Smetanova, M.: Bulletin of the Astronomical Institutes of Czechoslovakia 10, 192 (1959)

Popper, D.M.: Publications of the Astronomical Society of the Pacific 74, 129 (1962)

Popper, D.M.: The Astronomical Journal 169, 549 (1971)

Popper, D.M.: The Astrophysical Journal Supplement Series 71, 595 (1989)

Popper, D.M.: Annual review of astronomy and astrophysics 18(1), 115 (1980)

Prikhod'Ko, A.: Soviet Astronomy 5, 709 (1962)

Qian, S.: Astron. and Astrophys. Suppl. Ser. 146(3), 377 (2000)

Qian, S., Zhu, L., Boonrucksar, S.: Astronomy \& Astrophysics 396(2), 609 (2002)

Qian, S.: The Astronomical Journal 121(3), 1614 (2001)

Ramella, M., Giuricin, G., Mardirossian, F., Mezzetti, M.: Astrophysics and Space Science 71(2), 385 (1980)

Rhombs, C., Fix, J.: The Astrophysical Journal 216, 503 (1977)

Rhombs, C.G., Fix, J.D.: Acta Astronomica 26, 301 (1976)

Safar, J., Zejda, M.: Information Bulletin on Variable Stars 4888 (2000)

Samolyk, G.: Journal of the American Association of Variable Star Observers (JAAVSO) 26, 22 (1997)

Samolyk, G.: Journal of the American Association of Variable Star Observers (JAAVSO) 36, 171 (2008)

Samolyk, G.: JAAVSO 38, 85 (2010)

Samolyk, G.: JAVSO 39, 177 (2011)

Samus, N.N., Durlevich, O.V., et al.: VizieR Online Data Catalog 1 (2009) 
Sarna, M.J., De Greve, J.-P.: Quarterly Journal of the Royal Astronomical Society 37, 11 (1996)

Schaefer, B.E.: Journal of the American Association of Variable Star Observers (JAAVSO) 9, 45 (1980)

Shapley, H.: The Astrophysical Journal 38 (1913)

Shengbang, Q.: Astronomy and Astrophysics Supplement Series 146(3), 377 (2000)

Shengbang, Q.: Astrophysics and space science 282(2), 399 (2002)

Sitterly, B.: The Astrophysical Journal 53 (1921)

Smalley, B., Southworth, J., Pintado, O., Gillon, M., Holdsworth, D., Anderson, D., Barros, S., Cameron, A.C., Delrez, L., Faedi, F., et al.: Astronomy \& Astrophysics 564, 69 (2014)

Southworth, J., Pavlovski, K., Tamajo, E., Smalley, B., West, R.G., Anderson, D.: Monthly Notices of the Royal Astronomical Society 414(4), 3740 (2011)

Soydugan, E., İbanoğlu, C., Soydugan, F, Akan, M., Demircan, O.: Monthly Notices of the Royal Astronomical Society 366(4), 1289 (2006)

Srivastava, J., Kandpal, C.: Astrophysics and space science 209(1), 51 (1993)

Srivastava, R.: Astrophysics and space science 150(1), 173 (1988)

Srivastava, R., Padalia, T.: Astrophysics and Space Science 38(1), 79 (1975)

Strassmeier, K., Fekel, F.: Astronomy and Astrophysics 230, 389 (1990)

Strohmeier, W., Knigge, R.: Bamberg Veroeffentlichungen der Remeis-Sternwarte, Vol. 5, p. 10 (1961) 5, 10 (1961)

Strohmeier, W., Bauernfeind, H.: Bamberg Veroeffentlichungen der Remeis-Sternwarte, Vol. 7, p. 72 (1968) 7, 72 (1968)

Struve, O.: MNRAS 109, 487 (1948)

Struve, O.: Monthly Notices of the Royal Astronomical Society 109, 487 (1949)

Struve, O.: The Astrophysical Journal 106, 92 (1947)

Surkova, L.P., Svechnikov, M.A.: VizieR Online Data Catalog 5115 (2004)

Szczepanowska, A.: Acta Astronomica 6, 144 (1956)

Szczepanowska, A.: Acta Astronomica 9, 46 (1959)

Vesper, D., Honeycutt, K., Hunt, T.: The Astronomical Journal 121 (5), 2723 (2001)

Walter, K.: Astronomy and Astrophysics 5, 140 (1970)

Webbink, R., Hazen, M., Hoffleit, D.: Information Bulletin on Variable Stars 5298 (2002)

Whitney, B.: The Astronomical Journal 62, 371 (1957)

Whitney, B.: The Astronomical Journal 64, 258 (1959)

Wolf, M.: Astronomische Nachrichten 166, 77 (1904)

Wood, B., Forbes, J.: The Astronomical Journal 68, 257 (1963)

Woodsworth, A.W., Hughes, V.A.: Astronomy and Astrophysics 58, 105 (1977)

Wyse, A.B.: Lick Observatory Bulletin 17, 37 (1934)

Yoon, T.S., Honeycutt, R.K., Kaitchuck, R.H., Schlegel, E.M.: Publications of the Astronomical Society of the Pacific 106(697), 239 (1994)

Zakirov, M.: Information Bulletin on Variable Stars 4088 (1994)

Zakirov, M.: Astronomy Letters 22, 593 (1996)

Zakirov, M.: VizieR Online Data Catalog 902 (2001)

Zasche, P.: New Astronomy 16(3), 157 (2011)
Zasche, P., Liakos, A., Wolf, M., Niarchos, P.: New Astronomy 13(6), 405 (2008)

Zejda, M.: Information Bulletin on Variable Stars 5583 (2004)

Zejda, M.: Information Bulletin on Variable Stars 5287 (2002a)

Zejda, M.: Information Bulletin on Variable Stars 5287 (2002b)

Zhang, X., Luo, C., Fu, J.: The Astrophysical Journal 777(1), 77 (2013)

This manuscript was prepared with the AAS $\mathrm{ET}_{\mathrm{E}} \mathrm{X}$ macros v5.2. 